\title{
On the numerical integration of rate independent single crystal behavior at large strain
}

\author{
Mohamed Ben Bettaieb ${ }^{a, *}$, Olivier Débordes ${ }^{b}$, Abdelwaheb Dogui $^{c}$, Laurent Duchêne ${ }^{a}$, \\ Clément Keller ${ }^{\mathrm{d}}$ \\ ${ }^{a}$ ArGEnCo Department, $M S^{2} F$ Division, University of Liège, Chemin des Chevreuils 1, 4000 Liège, Belgium \\ ${ }^{\mathrm{b}}$ LMA E ECM, IMT, Technopole Château-Gombert, F13383 Marseille Cedex 13, France \\ ${ }^{\mathrm{c}}$ LGM, ENIM, 5019 Monastir, Tunisia \\ dErmeca-GPM, UMR 6634 CNRS - Université de Rouen, INSA de Rouen, avenue de l'université, 76800 Saint-Etienne du Rouvray, France
}

\section{A R T I C L E I N F O}

\section{Article history:}

Received 4 April 2011

Received in final revised form 26 October 2011

Available online 7 November 2011

\section{Keywords:}

Finite strain

FCC single crystal

Schmid's law

Non-linear hardening

Numerical integration

\begin{abstract}
A B S T R A C T
This paper presents a new numerical algorithm for the integration of the constitutive equations of a single crystal for finite rate-independent elastoplastic strains. The algorithm addressed in this paper is dedicated to face-centered-cubic (FCC) crystal structures. Its first feature is a much more efficient and more accurate integration scheme of the constitutive equations compared to previous attempts. This scheme is based on a fully implicit integration procedure, yet it may be transformed easily into an explicit scheme. Determining the set of active slip systems is performed by the use of a combinatorial search procedure, and the determination of the slip rates of the different active slip systems is based on the fixed point method. The second feature of this algorithm stems from the original method used to solve the ambiguity of the possible non-uniqueness of the set of active slip systems. A robust method, based on a small positive perturbation of the critical shear stresses, is proposed to overcome this difficulty. It is worth mentioning that the algorithm developed in this paper is not limited to one particular hardening law or to FCC crystal structures. Rather, it can be used and extended to various hardening laws and crystal structures (e.g. BCC or HCP...) in a straightforward manner. The authors demonstrate the performance of the proposed algorithm and illustrate its accuracy and efficiency through various numerical simulations at the single crystal and polycrystal scales. The predicted results obtained from those simulations were compared with those obtained using other numerical techniques and algorithms (i.e., a pseudo-inversion technique and an explicit algorithm). Our numerical predictions are also compared with some numerical and experimental results from other papers. The response of the polycrystal was computed by using the proposed algorithm combined with Taylor's homogenization scheme, which is used to compute the overall polycrystalline behavior. The paper ends with a statistical study of the influence of the perturbation technique on the response prediction for a single crystal and a polycrystal.
\end{abstract}

(c) 2011 Elsevier Ltd. All rights reserved.

\section{Introduction}

Crystal plasticity has attracted much attention due to its ability to relate the plastic behavior of crystalline materials to their microstructures. The mechanical modeling of single crystal behavior, at a finite strain range, is now reasonably well

\footnotetext{
* Corresponding author.

E-mail address: Mohamed.BenBettaieb@ulg.ac.be (M. Ben Bettaieb).
} 
understood and fairly well established (Asaro, 1983; Hill and Rice, 1972; Mandel, 1965; Taylor and Elam, 1923. . ). However, numerical modeling remains a controversial subject especially with the development of finite element codes. These codes are generally used to model and simulate the mechanical behavior of metallic components and structures or to predict the overall behavior of the crystal aggregate (by using some homogenization techniques). In some special cases (for example, the $\mathrm{FE}^{2}$ method), the size of the problems and structures is rather large and requires a great deal of CPU and memory space. Therefore, developing numerical integration algorithms with improved efficiency, speed and accuracy has become more desirable. In the rate independent formulation of single crystal behavior at finite strains, there have been two long-standing problems:

- The first one is how to integrate the set of constitutive equations. In this field, this set of equations is known to be strongly non-linear and this non-linearity has two distinct sources: the first comes from the material behavior and is related to the expression of the hardening law (when hardening is considered). Indeed, the hardening laws used in the literature generally assume a complicated, non-linear evolution of the rates of the various critical shear stresses. These laws were developed differently by particular selection of the primary variables (slip rates in Ben Bettaieb, 2006; Chang and Asaro, 1981; Kuchnicki et al., 2006; Ling et al., 2005; Miehe and Schröder, 2001; Miehe et al., 1999; Krishna Kanjarla et al., 2010 and critical shear stresses in Anand and Kothari, 1996; Knockaert et al., 2000; Kothari and Anand, 1998). The second source of non-linearity is geometrical and is related to the evolution of the crystal lattice's rotation (under the finite strain assumption). In order to solve this set of non-linear equations, several numerical algorithms have been developed and tested. Typically, these algorithms reach the solution incrementally and can be classified into two main families: explicit algorithms based on a forward Euler scheme and implicit algorithms based on a backward Euler scheme. A third, smaller, intermediate family, in between implicit and explicit algorithms, is called semi-implicit algorithms.

- Explicit integration algorithms: Developing of explicit algorithms is known to be relatively straightforward, but it generally requires very short time or loading increments to avoid numerical instabilities. In this explicit family (NematNasser and Amirkhizi, 2007; Anand and Kothari, 1996; Ben Bettaieb, 2006; Knockaert et al., 2000; Kuchnicki et al., 2006; Maniatty et al., 1992; Mathur and Dawson, 1989; Peirce et al., 1982; Pourboghrat et al., 2007; Rossiter et al., 2010), the critical shear stresses and the rotation are assumed to be constant over the time increment and chosen to be equal to their values at the beginning of the time step. Choosing of this class of algorithms allows the execution time to be reduced (because it is not necessary to use an iterative procedure to compute the slip rates of the different slip systems) at each time increment, but it requires the use of a very large number of strain increments.

- Implicit integration algorithms: The implementation of implicit schemes is more complicated than for explicit ones but it ensures higher accuracy and unconditional stability, which allows for large time steps and provides a dramatic improvement in the convergence rate. In this implicit family, the critical shear stresses and the rotation of the crystal lattice are unknown, producing a set of nonlinear equations. This set has to be solved by traditional iterative methods (generally those such as the Newton-Raphson procedure or the fixed point method).

- Semi-implicit integration algorithms: In this class of algorithms, the crystal lattice's rotation is evaluated at the beginning of the time increment. However, the computation of the critical shear stresses is based on an implicit scheme. So this algorithm class is implicit in the hardening rates, and explicit with regards to the increment of lattice rotation. This semi-implicit scheme is based on an iterative resolution of the consistency conditions (McGinty and McDowell, 2006; Watanabe et al., 2010) or a linearization of the hardening law (Ben Bettaieb, 2006; Débordes et al., 2005) over each time step.

The purpose of our paper is to develop a fully implicit algorithm to integrate the constitutive equations of the single crystal at rate-independent elastoplastic finite strains. Even though it is applied here to FCC crystals, this algorithm is general enough to be used for any crystal structure and any hardening law. From a mechanical viewpoint, this algorithm is based on the consistency condition (derived from Schmid's law), which can be expressed mathematically by a non-linear complementarity relation between two positive variables, namely the slip rate and the difference between the rate of the critical shear stress and the rate of the resolved shear stress. This condition can be considered to be a non-linear complementarity problem (NLCP). This NLCP is resolved numerically by a combinatorial search procedure (in order to identify the set of the active slip systems) and by the fixed point method (in order to determine the slip rate of those systems). This implicit algorithm can be transformed easily into an explicit one by avoiding the use of the fixed point method in order to update the slip rates. To the author's knowledge, the development of a fully implicit scheme for the integration of the constitutive equations of a single crystal for finite rate-independent elastoplastic strains has not been undertaken in the scientific literature. More details about the originalities of the developed implicit algorithm compared to the algorithms presented in the literature are exposed in Section 4.8 .

- The second long-standing problem in the rate independent formulation of single crystal behavior concerns the method used in order to solve the well-known ambiguity of the non-uniqueness of the active slip systems set and the corresponding slip rates. This ambiguity occurs in some cases of strain paths and when the stress state is situated at degenerated sides of the yield surface (i.e. when the slip systems are interdependent). The literature provides several contributions that try to circumvent this difficulty. These contributions can be classified into two distinct families: the first is based 
on physical or thermodynamic considerations and the second on a purely numerical regularization of this ambiguity (without any physical motivation).

- Physical solutions: We mainly cite (Taylor and Elam, 1923), who selected the solution, that minimizes the derivative of the plastic work. Hamelin et al. (2011) developed a first-order selection criterion, based on the minimization of the change of the internal work with respect to the von Mises strain. Earlier (Renouard and Wintenberger, 1981) proposed a method to select the combination of the active set of slip systems based on the fact that some combinations rotate towards orientations which need less internal work dissipation than others. This strategy is followed by Driver et al. (1984), Fortunier and Driver (1987) and Skalli et al. (1985) in order to simulate the behavior of aluminum single crystals. Franciosi and Zaoui (1991) used a minimizing hardening criterion in order to select the set of active slip systems. Many authors have applied a rate-dependent formulation based on power-type creep laws without differentiation of slip systems into active and inactive sets via a loading function (Asaro and Needleman, 1985; Mathur and Dawson, 1989; Peirce et al., 1982, 1983). Finally (Schmidt-Baldassari, 2003) used an augmented Lagrangian formulation based on the principle of the maximum dissipation in order to avoid the non-uniqueness ambiguity.

- Numerical solutions: we cite in this category the work of Van Houtte (1981), who randomly selected a solution. Arul Kumar et al. (2010) used a postulate based on a mathematical manipulation of the consistency condition. Another strategy followed by Anand and Kothari (1996) and Knockaert et al. (2000), consists in using the pseudo-inversion technique (this is equivalent to projecting, in some mathematical sense, the deformation rate on the set of positive slip rates). Miehe and Schröder (2001) and Schröder and Miehe (1997) proposed an alternative general inverse where the reduced space is obtained by dropping columns of the local Jacobian associated with zero diagonal elements within a standard factorization procedure. Many other authors (Gambin, 1991, 1992; Montheillet et al., 1985; Toth et al., 1991; Van Houtte, 1987) proposed a rate-independent approach based on the concept of crystals with smooth yield surface. In this approach, only one yield function is used in order to calculate the crystal spin and the incremental shear strains on the active slip systems. Therefore, the slip system ambiguity in crystal plasticity is avoided. Elbououni (2002) and Elbououni et al. (2003) developed a technique to remove this ambiguity, based on the postulate of minimum plastic spin (in the case of plane stress problems). More recently Zamiri and Pourboghrat developed in Zamiri and Pourboghrat (2010) a new technique, based on the so-called combined constraints crystal plasticity (CCCP) model, to solve this ambiguity.

In this contribution, the loss of uniqueness is overcome by adding a slight, positive perturbation to the critical shear stresses. This perturbation is different from one system to another and can be interpreted as a result of a material imperfection or a previous weak latent hardening. The application of this technique allows the degenerated sides to be broken down into simple ones and radically eliminates the non uniqueness ambiguity. It must be noted that some researchers demonstrate that this loss of uniqueness is absent in real material due to anisotropic hardening of the slip systems (e.g. Peeters et al., 2001).

The paper is organized as follows:

- Section 2 outlines the constitutive equations for single crystal plasticity for the case of rate-independent plasticity and finite strain.

- Section 3 details the FCC single crystal's yield surface topology under the plane stress assumption and in the general 3-D case.

- Section 4 provides the algorithmic developments in an incremental formulation. The details of the implicit integration scheme are outlined.

- The performance of the developed algorithm is studied in Section 5. In this section, the sensitivity of the mechanical response of the single crystal and the polycrystal to the perturbation technique is also analyzed.

\subsection{Notations}

The derivations presented in this paper are carried out using classic conventions. The assorted notations can be combined between them. Additional notations will be clarified as needed following related equations.

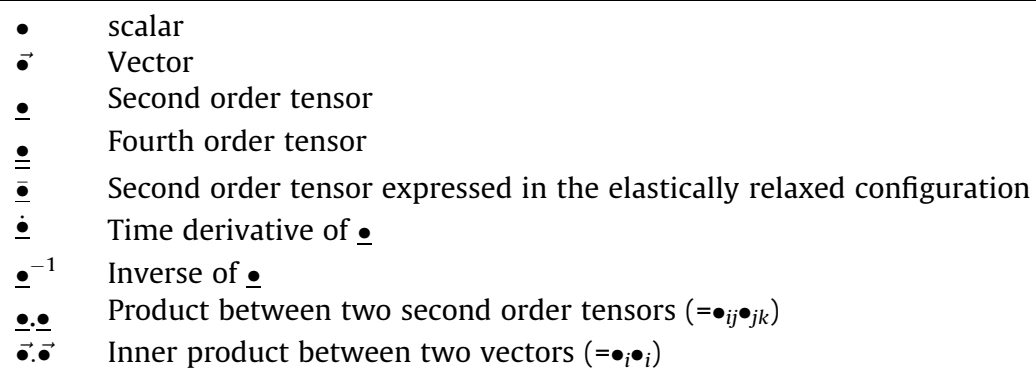


$\vec{\bullet} \otimes \vec{\bullet}$ Tensorial product between two vectors $\left(=\bullet_{i} \bullet_{j}\right)$

$\bullet:$ Double contraction between two second order tensors $\left(=\bullet_{i j} \bullet_{i j}\right)$

: $\bullet$ Double contraction between fourth and second order tensors $\left(=\bullet_{i j k l} \bullet_{k l}\right)$

$\bullet^{t} \quad$ Tensor $\bullet$ at time $t$ (for convenience, the dependence on time presented by superscript $t$ is automatically omitted when the quantity is constant over the time increment)

$\bullet^{T} \quad$ Transpose of tensor $\bullet$

$(\bullet)_{S} \quad$ Symmetric part of tensor $\bullet$

$(\bullet)_{A} \quad$ Skew-symmetric part of tensor $\bullet$

(i) The evaluation of $\bullet$ in the $i$ th iteration

\section{Constitutive equations}

\subsection{Decomposition of the velocity gradient}

The classic treatment of finite plasticity deformations may be traced back to several early works (such as Asaro, 1983; Hill and Rice, 1972; Mandel, 1965; Taylor and Elam, 1923). It is based on the assumption of the existence of an infinite number of elastically relaxed configurations (i.e., configurations obtained by elastic unloading to a stress free state), which are defined up to an arbitrary rotation $\underline{r}$ with respect to the initial one. To satisfy the objectivity principle (Dogui, 1989), the constitutive equations may be written in one of these elastically relaxed configurations. Thus, we denote all the tensors and vectors evaluated in this configuration by a superposed bar. In the case of single crystals, the rotation is chosen such that the orientation tensors $\bar{M}_{s}$ of the different slip systems (see Appendix A for the definition of $\bar{M}_{s}$ for the case of FCC single crystals) remain unchangeable during the deformation.

The FCC single crystal considered is submitted to a velocity gradient $\underline{l}$. The transport $\underline{\underline{l}}$ of $\underline{l}$ in the elastically relaxed configuration is split into a symmetric part $\underline{\bar{d}}$ and a skew-symmetric part $\underline{\bar{w}}$. Assuming that the elastic strain is very small compared to the plastic strain, $\underline{\underline{l}}$ can be broken down additively into an elastic component and a plastic one. The elastic component of $\underline{\bar{l}}$ can also be written as the sum of the elastic strain rate $\underline{\bar{d}}^{e}$ and $\underline{r}^{T} \underline{\dot{r}}$. However the plastic component of $\underline{\underline{l}}$ can be written as the sum of the plastic strain rate $\underline{\bar{d}}^{p}$ and the plastic spin $\underline{\bar{w}}^{p}$. The elastic component describes the lattice distortion and the plastic one is solely due to the contribution of the slip rates on the various oriented slip systems. With these notations, the basic kinematic relations can be written as:

$$
\underline{\underline{l}}=\underline{r}^{T} \underline{l} \underline{r}=\underline{\bar{d}}+\underline{\bar{w}} ; \quad \underline{\bar{d}}=\underline{\bar{d}}^{e}+\underline{\bar{d}}^{p} ; \quad \underline{\bar{w}}=\underline{r}^{T} \underline{\underline{r}}+\underline{\bar{w}}^{p} ; \quad \underline{\bar{d}}^{p}+\underline{\bar{w}}^{p}=\sum_{s=1}^{12} \dot{\gamma}_{s}^{*} \bar{M}_{s},
$$

where $\dot{\gamma}_{s}^{*}$ is the algebraic value of the slip rate of the slip system $s$.

The evolution of the rotation $\underline{r}$ is derived from the third equation of (1):

$$
\underline{r}^{T} \underline{\dot{r}}=\underline{\bar{w}}-\underline{\bar{w}}^{p} \text {. }
$$

To avoid the use of absolute value and sign functions, each slip system is split into two oriented and opposite oriented slip systems (see Appendix A). With this new definition, the last relation of Eq. (1) becomes

$$
\underline{\bar{d}}^{p}+\underline{\bar{w}}^{p}=\sum_{s=1}^{24} \dot{\gamma}_{s} \underline{\bar{M}}_{s} ; \quad \dot{\gamma}_{s} \geqslant 0 \quad(s=1, \ldots, 24) .
$$

\subsection{Elastoplastic behavior}

The elastic part of the behavior law is classically written as:

$$
\dot{\bar{\sigma}}=\underline{\bar{C}}: \overline{\bar{d}}^{e}
$$

where $\underline{\underline{C}}$ is the fourth order elasticity tensor. Here linear isotropic elasticity is assumed.

In ad̄ dition, the plastic component of the behavior law is defined by Schmid's law (Schmid and Boas, 1935). This law states that slip occurs when the resolved shear stress $\tau_{s}$ on a slip system $s$ exceeds a critical value $\tau_{s}^{c}$ :

$$
\forall s=1, \ldots, 24:\left\{\begin{array}{l}
\tau_{s}<\tau_{s}^{c} \Rightarrow \dot{\gamma}_{s}=0 \\
\tau_{s}=\tau_{s}^{c} \Rightarrow \dot{\gamma}_{s} \geqslant 0
\end{array}\right.
$$


where the resolved shear stress $\tau_{s}$ is defined as the projection of the Cauchy stress $\underline{\bar{\sigma}}$ onto the slip system $s$ and is equal to $\overline{\bar{\sigma}}: \bar{M}_{s}$.

\subsection{Hardening law}

The hardening law describes the evolution of the critical shear stress $\tau_{s}^{c}$ during the loading history. The literature provides several models of the hardening law which are generally motivated by the crystal's physical microstructure and dependent, via a hardening modulus $h$, on the slip rate of the different slip systems. These hardening laws can be classified into two main families: stress-based and strain-based hardening laws.

In the case of the stress based hardening laws, the primary variables of the hardening modulus $h$ are the critical shear stresses (Eq. (6)). However for the case of strain based hardening laws, the primary variables are the accumulated slip of the different slip systems as mentioned in the Eq. (7):

$$
\begin{array}{ll}
\forall s=1, \ldots, 12: & \dot{\tau}_{s}^{c}=\dot{\tau}_{s+12}^{c}=\sum_{g=1}^{g=12} h_{s g}\left(\tau_{g}^{c}\right)\left(\dot{\gamma}_{g}+\dot{\gamma}_{g+12}\right), \\
\forall s=1, \ldots, 12: & \dot{\tau}_{s}^{c}=\dot{\tau}_{s+12}^{c}=\sum_{g=1}^{g=12} h_{s g}\left(\gamma_{g}\right)\left(\dot{\gamma}_{g}+\dot{\gamma}_{g+12}\right) .
\end{array}
$$

The Voce law is the result of the second form of the hardening law (Eq. (7)). It is very similar to the law used by Abdul-Latif et al. (1998).

Eqs. (6) and (7) can be written commonly in the following generic form:

$$
\forall s=1, \ldots, 12: \quad \dot{\tau}_{s}^{c}=\dot{\tau}_{s+12}^{c}=\sum_{g=1}^{g=12} h_{s g}\left(\dot{\gamma}_{g}+\dot{\gamma}_{g+12}\right),
$$

where $h$ is dependent on the critical shear stresses or the accumulated slip for the stress or the strain-based hardening laws respectively. This hardening modulus is defined by several forms in the literature: diagonal, isotropic, anisotropic, symmetric or asymmetric. A complete compilation of the most important hardening laws for single crystals is given in Appendix B. In the present paper, the integration scheme is general enough to be independent of the choice of the hardening law.

\section{Topology of the yield surface in the case of FCC single crystals}

In this section, the topology of the yield surface of FCC single crystal is established under the plane stress assumption and for the general 3D case. In these two cases, the yield surface is defined when the critical stresses are identical for the different slip systems and when the critical shear stresses differ from one system to another (e.g., as a result of a slight, random perturbation of the critical shear stresses). The description of this topology is required in order to introduce the perturbation technique which is used in the algorithmic part of this paper.

\subsection{FCCP single crystal}

The FCCP (the plane FCC) is a particular case of the 3D FCC single crystal submitted to a plane loading path in a symmetry plane (Boukadia, 1988; Boukadia and Sidoroff, 1988; Chenaoui, 1992; Chenaoui et al., 2000; Elbououni, 2002; Elbououni et al., 2003). Boukadia and Sidoroff (1988) demonstrated, in this case, that the 12 "physical" slip systems are combined to define an equivalent single crystal with 5 pseudo-slip systems. If the critical shear stresses are identical for all systems, the yield locus is a polyhedron defined by 10 facets, 20 edges and 12 vertices. Among these 12 vertices, 8 are simple (intersection of 3 facets) and 4 are degenerated (intersection of 4 facets). After the perturbation of the different critical shear stresses and regardless of the value of this perturbation, the new yield locus becomes a polyhedron at 16 vertices (all simple). In this case, the initial degenerated vertices are split into edges: $A$ into $A^{\prime} A^{\prime \prime}$ which leads to the disappearance of degenerations. The same decomposition is applied on the other degenerated vertices. Fig. 1 illustrates the topology of the yield surface of the FCCP single crystal before and after perturbation.

\subsection{D FCC single crystal}

As in the case of the majority of metals, the plastic deformation is assumed to be isochoric. So the yield locus (the set of all deviatoric stresses satisfying Schmid's law) can be presented in the linear five-dimensional deviatoric space (in the case of three-dimensional symmetric tensors). In this space, the yield locus is a closed convex polyhedron and is made of the intersection of 24 half-spaces defined by 24 hyper-planes. For each side of the boundary of this yield locus, let $i$ be the dimension of the smallest affine subspace containing this side (in a linear space, an affine subspace is the translation of a linear subspace). This integer $i$ ranges from 0 (if the side is a vertex) to 4 (if the side is a face). An $i$-side (i.e. a side with dimension $i$ ) is the intersection of at least (5-i) hyper-planes, and it is called degenerated when it is the intersection of more than 
a

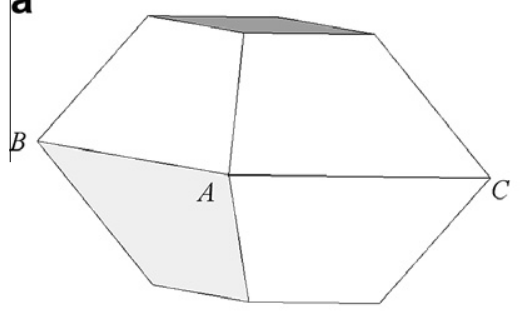

b

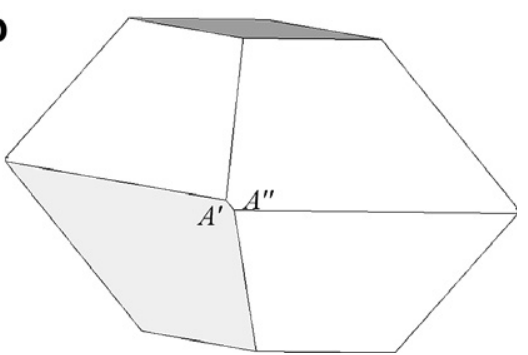

Fig. 1. Topology of the F.C.C.P2 yield locus (Boukadia and Sidoroff, 1988): (a) before perturbation and (b) after perturbation.

Table 1

Degeneration of the three-dimensional single crystal yield locus before and after perturbation.

\begin{tabular}{lll}
\hline & Before perturbation & After perturbation \\
\hline Number of vertices $(\boldsymbol{O}$-sides) & 56 (all degenerated) & 204 (all simple) \\
Number of 1 -sides & 216 (72 are degenerated) & 510 (all simple) \\
Number of 2 -sides & $270(30$ are degenerated) & 452 (all simple) \\
Number of3-sides & 132 (all simple) & 168 (all simple) \\
Number of 4 -sides & 24 (all simple) & 24 (all simple) \\
\hline
\end{tabular}

(5-i) hyper-planes, otherwise it is called simple. When the critical shear stresses are identical for all slip systems, it is widely known (Bishop and Hill, 1951a,b) that all the 0-sides are degenerated. The first column of Table 1 gives more indications about the degeneration state of the other sides in this case; it shows that this degeneration is a very common case.

\section{Algorithmic developments}

\subsection{Incremental algorithm}

The time integration of the evolution problem composed by Eqs. (1)-(5) and (8) proceeds by discretizing the deformation history in time and numerically integrating these equations over each typical time step $I^{\Delta}=\left[t_{0}, t_{0}+\Delta t\right]$. For this purpose, we assume the material parameters (elasticity parameters, hardening parameters) and the subsequent quantities are known: $\underline{\sigma}^{t_{0}}, \underline{r}^{t_{0}}, \gamma_{s}^{t_{0}}$ and $\tau_{s}^{c t_{0}}$ (for $s=1, \ldots, 24$ ) at $t_{0}$. The aim of the incremental algorithm is to compute $\underline{\sigma}^{t_{0}+\Delta t}, \underline{r}^{t_{0}+\Delta t}, \gamma_{s}^{t_{0}+\Delta t}$ and $\tau_{s}^{c t_{0}+\Delta t}$ (for $s=1, \ldots, 24$ ) at $t_{0}+\Delta t$. The time step $I^{\Delta}$ is divided into several sub-steps or sub-increments $I^{\delta}$ over which Schmid's criterion is fulfilled. The length $\delta t$ of each sub-increment $I^{\delta}$ is computed by the following algorithm (described in Sections 4.2 , $4.3,4.4$ and 4.5 ). In order to simplify the notation, the subscript $t_{0}+\delta t$ will be dropped in the following developments, with the understanding that all variables are evaluated at $t_{0}+\delta t$, unless otherwise indicated.

\subsection{Identification of the mechanical behavior over the sub-increment $I^{\delta}$}

The aim of this step is to identify the mechanical behavior over the sub-increment $I^{\delta}$. With this aim, let us introduce the set of potentially active slip systems $P^{t_{0}}$ at $t_{0}$ :

$$
P^{t_{0}}=\left\{s=1, \ldots, 24 ; \underline{\bar{M}}_{s}: \underline{\bar{\sigma}}^{t_{0}}=\tau_{s}^{c t_{0}}\right\} .
$$

At this level, two possibilities can occur:

- If (a) $P^{t_{0}}=\varnothing$ or (b) $P^{t_{0}} \neq \varnothing$ and $\underline{M}_{g}: \underline{\bar{d}} \leqslant 0$ for all $g \in P^{t_{0}}$ then the mechanical behavior is purely elastic over $I^{\delta}$. This case is studied in Subsection 4.3 .

- If $P^{t_{0}} \neq \varnothing$ and $\bar{M}_{g}: \underline{\bar{d}}>0$ for at least one $g \in P^{t_{0}}$ then the mechanical behavior is elastic-plastic over $I^{\delta}$. This case is studied in Subsection 4.4 .

In spite of multiple similarities between the algorithmic treatments of the two possibilities, the two cases are studied separately (Sections 4.3 and 4.4.) for the sake of clarity and consistency of the presentation.

\subsection{Elastic case}

The first aim of this elastic algorithm is to compute the length $\delta t$ of the sub-increment $I^{\delta}$ over which the behavior remains purely elastic. The second aim is to update the different mechanical variables at $t_{0}+\delta t$. This computation is carried out using an iterative procedure (based on the fixed point method): 


\section{Step 1}

- Initialize: $j=0, \delta t^{(0)}=\Delta t$ and $r^{(0)}=r^{t_{0}}$

- Set $j=1$.

\section{Step 2}

For $(j>0)$ do the following computations:

- compute $\underline{r}^{(j)}$ as follows:

$$
\underline{r}^{(j)}=e^{\delta t^{(j-1)} \underline{\bar{w}}^{(j-1)}} \underline{r}^{t_{0}} ; \quad \underline{\underline{w}}^{(j-1)}=\underline{r}^{(j-1) T} \underline{w r}^{(j-1)} .
$$

This equation is the result of the implicit integration of Eq. (2) over $\left[t_{0}, t_{0}+\delta t\right]$ (see Simo and Vu-Quoc, 1986).

- compute $\underline{\bar{d}}^{(j)}$ as follows:

$$
\underline{\bar{d}}^{(j)}=r^{(j) T} \underline{d} \underline{r}^{(j)}
$$

- compute $\delta t^{(j)}$ as follows $\left(\delta t^{(j)}\right.$ must, of course, be greater than 0$)$ :

$$
\delta t^{(j)}=\min \left\{\Delta t, \frac{\tau_{g}^{c t_{0}}-\underline{\bar{M}}_{g}: \underline{\bar{\sigma}}^{t_{0}}}{\underline{\bar{M}}_{g}: \underline{\bar{C}}: \underline{\bar{d}}^{(j)}} ; \quad \forall g \in\{1, \ldots, 24\}\right\} .
$$

\section{Step 3}

The convergence of this iterative procedure occurs when the difference between $\delta t^{(j)}$ and $\delta t^{(j-1)}$ becomes smaller than a specified tolerance $\varepsilon$ (typically chosen to be equal to $10^{-5}$ ):

$$
\left|\delta t^{(j)}-\delta t^{(j-1)}\right|<\varepsilon
$$

If condition (13) is verified, then $\delta t^{(j)}$ is the correct length $\delta t$ of the sub-increment $I^{\delta}$, then go to Step 4 . Otherwise set $j \leftarrow j+1$ and go to Step 2 .

\section{Step 4}

Update the problem variables and go to the next sub-increment:

$$
\begin{aligned}
& \underline{r}^{t_{0}} \Leftarrow \underline{r}^{t_{0}+\delta t} \quad ; \quad \underline{\bar{\sigma}}^{t_{0}} \Leftarrow \underline{\bar{\sigma}}^{t_{0}+\delta t}=\underline{\bar{\sigma}}^{t_{0}}+\delta t(\underline{\underline{\bar{C}}}: \underline{\bar{d}}) \quad ; \quad \gamma_{g}^{t_{0}} \Leftarrow \gamma_{g}^{t_{0}} \quad ; \quad \tau_{g}^{c t_{0}} \Leftarrow \tau_{g}^{c t_{0}} \\
& \Delta t \Leftarrow \Delta t-\delta t \quad ; \quad t_{0} \Leftarrow t_{0}+\delta t \text {. }
\end{aligned}
$$

The rotation $\underline{r}^{t_{0}+\delta t}$ and the strain $\underline{\bar{d}}$ are equal to the last converged values computed by Eqs. (10) and (11) respectively. As the behavior is purely elastic, the accumulated slip and the critical shear stress of the different slip systems remain constant over $I^{\delta}$.

\subsection{Plastic case}

\subsubsection{Non linear complementarity problem}

In the plastic case, the set $P^{t_{0}}$ is different from $\varnothing$. Each element of $P^{t_{0}}$ must fulfill the consistency condition based on Schmid's law:

$$
\forall s \in P^{t_{0}}, \quad \forall t \in I^{\delta}: \quad \dot{\gamma}_{s}^{t} \geqslant 0 ; \quad\left(\dot{\tau}_{s}^{c t}-\dot{\tau}_{s}^{t}\right) \geqslant 0 ; \quad \dot{\gamma}_{s}^{t}\left(\dot{\tau}_{s}^{c t}-\dot{\tau}_{s}^{t}\right)=0 .
$$

A slip system is said active only when its slip rate is strictly positive. Knowing that only the systems belonging to $P^{t_{0}}$ may be active, $\dot{\tau}_{s}^{c t}$ and $\dot{\tau}_{s}^{t}$ can be written as:

$$
\forall s \in P^{t_{0}}, \quad \forall t \in I^{\delta}:\left\{\begin{array}{l}
\dot{\tau}_{s}^{c t}=\frac{\partial \tau_{s}^{c t}}{\partial t}=\sum_{g \in P^{t_{0}}} h_{s g}^{t} \dot{\gamma}_{g}^{t}, \\
\dot{\tau}_{s}^{t}=\underline{\bar{M}}_{s}: \underline{\dot{\bar{\sigma}}}^{t}=\bar{M}_{s}: \underline{\bar{C}}: \underline{\bar{d}}^{t}-\sum_{g \in P^{t_{0}}} \dot{\gamma}_{g}^{t}\left(\overline{\bar{M}}_{s}: \underline{\underline{C}}: \bar{M}_{g}\right) .
\end{array}\right.
$$

After a few straightforward algebraic manipulations, the substitution of (16) into (15) leads to:

$$
\forall s \in P^{t_{0}}, \quad \forall t \in I^{\delta}: \quad \dot{\gamma}_{s}^{t} \geqslant 0 ; \quad\left(\varphi_{i(s)}^{t}=\sum_{g \in P^{t_{0}}}\left(\Psi_{i(s) i(g)}^{t} \dot{\gamma}_{g}^{t}-Z_{i(s)}^{t}\right)\right) \geqslant 0 ; \quad \varphi_{i(s)}^{t} \dot{\gamma}_{s}^{t}=0,
$$

where $i(g)$ refers to the rank of the $g$ th system in $P^{t_{0}}$. The components of the square matrix $\Psi^{t} \in R^{\operatorname{card}\left(P^{t_{0}}\right) \times \operatorname{card}\left(P^{\left.t_{0}\right)}\right.}$ and the vector $Z^{t} \in R^{\operatorname{card}\left(P^{\left.t_{0}\right)}\right.}$ are:

$$
\forall g, s \in P^{t_{0}}: \quad \Psi_{i(s) i(g)}^{t}=\underline{\bar{M}}_{s}: \underline{\underline{C}}: \underline{\bar{M}}_{g}+h_{s g}^{t} ; \quad Z_{i(s)}^{t}=\underline{\bar{M}}_{s}: \underline{\bar{C}}: \underline{\bar{d}}^{t} .
$$

At any time $t \in I^{\delta}$, Eq. (17) is an NLCP, and it is identically equivalent to the subsequent optimization problem:

$$
\text { Find }\left\{\dot{\gamma}_{s}^{t}\right\}_{s \in P^{t_{0}}} \text { such that } \min \left\{\sum_{s \in P^{t_{0}}} \dot{\gamma}_{s}^{t} \varphi_{i(s)}^{t} ; \forall s \in P^{t_{0}}: \dot{\gamma}_{s}^{t} \geqslant 0, \varphi_{i(s)}^{t} \geqslant 0\right\}=0 \text {. }
$$


The last equation shows that the integration of the evolution problem composed of Eqs. (1)-(5) and (8) can be reduced to the determination of the slip rates of the various potentially active systems.

\subsubsection{Existence and uniqueness of the solution of the NLCP}

In spite of the large number of scientific works dedicated to the study of the NLCP problem (Chitra and Subrahmanyam, 1987; Cottle, 1966; Karamardian, 1969a,b; Kyparisis, 1986; Megiddo and Kojima, 1977), the existence and uniqueness conditions of a solution remain ambiguous for our purpose. However these conditions are clearer in the case of the LCP (linear complementarity problem):

- Existence: According to Golub and Van Loan (1983), the solution of the LCP exists when $Z^{t} \in R^{\text {card }\left(P^{t_{0}}\right)}$ (which is the case here).

- Uniqueness: According to Hill and Rice (1972), a positive definite matrix $\Psi^{t}$ leads to a unique solution of (19). If $\Psi^{t}$ is singular, the number of solutions may be infinite and the set of slip rates are not necessarily unique.

It is worth noting that the NLCP (19) is transformed into a LCP when the matrix $\Psi^{t}$ and the vector $Z^{t}$ are independent from the slip rates $\dot{\gamma}_{s}^{t}$ (which is the case of explicit integration schemes).

From our numerical examples, we noted that a solution to the NLCP (19) was always found. This solution is unique when the matrix $\Psi^{t}$ is positive definite. However, when this matrix is singular or when at least one of its eigenvalues is negative, the uniqueness of the solution of (19) is not guaranteed.

\subsubsection{Plastic integration algorithm}

The first aim of this plastic integration algorithm is to solve the NLCP of Eq. (19). This solution is based on the identification of the active slip system set by using a combinatorial research procedure and on the computation of the slip rates of the different active systems by using the fixed point method (Appendix C explains this method and its characteristics).

The second aim is to compute the length of the sub-increment $I^{\delta}$, over which Schmid's law remains fulfilled. The third aim is to update the mechanical variables at $t_{0}+\delta t$. Below, the steps used in this implicit plastic algorithm are described.

Step 1 The aim of this step is to identify the set of the active slip systems from the set of the potentially active slip systems $P^{t_{0}}$ by using a combinatorial research procedure. We assume here that this set of active systems remains unchanged over $I^{\delta}$.

\section{Sub-step 1.1}

- We start the first combination by assuming that the active set of slip systems $A$ coincides with the potentially active ones: $A=P^{t_{0}}$.

\section{Sub-step 1.2}

- Assume that $\dot{\gamma}_{s}^{t_{0}}$ is equal to 0 for the systems $s$ belonging to $P^{t_{0}} \backslash A$ :

$\forall s \in P^{t_{0}} \backslash A: \quad \dot{\gamma}_{s}^{t_{0}}=0$.

- The slip rates of the systems belonging to the set $A$ are computed by extracting the matrix $\widetilde{\Psi}^{t_{0}}$ from $\Psi^{t_{0}}$ and $\widetilde{Z}^{t_{0}}$ from $Z^{t_{0}}$ at time $t_{0}$ and by enforcing $\dot{\tau}_{s}^{c t_{0}}-\dot{\tau}_{s}^{t_{0}}=0(s \in A)$ :

$\forall s, g \in A: \quad \widetilde{\Psi}_{i(g) i(s)}^{t_{0}} \dot{\gamma}_{s}^{t_{0}}=\widetilde{Z}_{i(g)}^{t_{0}}$.

If the matrix $\widetilde{\Psi}^{t_{0}}$ is singular, we use the perturbation technique. This technique modifies the data slightly and leads to an elastic case. This is the result of the strict positivity of the perturbation value. Therefore, we follow the algorithm used in Section 4.3 relative to the elastic case.

\section{Sub-step 1.3}

- If the set $\dot{\gamma}_{s}^{t_{0}}(s \in P)$, computed by Eqs. (20) and (21), satisfies the first and second conditions of Eq. (17) at time $t_{0}$, then $A$ is a set of active slip systems, which is chosen, and we go to Step 2. Otherwise we choose another set $A$ and start over with Sub-step 1.2.

Step 2 The aim of this step is to compute the slip rates of the different active slip systems and the length $\delta t$ of $I^{\delta}$, the set of active slip systems being determined in the previous step.

\section{Sub-step 2.1}

- Initialize $j=0, \delta t^{(0)}=\Delta t$.

- Set $j=1$.

- For $(j>0)$ do the following sub-steps: 


\section{Sub-step 2.2}

- Compute the slip rates of the systems belonging to the set $A$ by extracting the matrix $\widetilde{\Psi}$ from $\Psi$ and $\widetilde{Z}$ from $Z$ and by enforcing $\dot{\tau}_{s}^{c}-\dot{\tau}_{s}=0(s \in A)$ :

$\forall s, g \in A: \quad \widetilde{\Psi}_{i(g) i(s)} \dot{\gamma}_{s}=\widetilde{Z}_{i(g)}$.

The $\widetilde{\Psi}$ component and $\widetilde{Z}$ are strongly dependent on the value of $\dot{\gamma}_{s}(s \in A)$ (see Eq. (16)). Indeed $\widetilde{\Psi}$ depends on the components of $h_{g s}$ which depends directly (in the case of a strain-based hardening law) or indirectly (in the case of a stress-based hardening law) on $\dot{\gamma}_{s}$. However, the vector $\widetilde{Z}$ depends on the rotation $\underline{r}$, which, in turn, depends on $\dot{\gamma}_{s}$ (see Eq. (2)). So the system of Eq. (22) is nonlinear. Its resolution is carried out as explained in Section 4.5 by using the fixed point method.

\section{Sub-step 2.3}

Deduce the rates $\dot{\tau}_{g}^{c}$ and $\dot{\tau}_{g}(g \in\{1, \ldots, 24\})$ from Eqs. (1), (4) and (8):

$$
\begin{aligned}
& \underline{\bar{d}}^{p}+\underline{\bar{w}}^{p}=\sum_{s \in A} \dot{\gamma}_{s} \underline{\bar{M}}_{s} ; \quad \underline{\dot{\bar{\sigma}}}=\underline{\bar{C}}:\left(\underline{\bar{d}}-\underline{\bar{d}}^{p}\right), \\
& \forall g \in\{1, \ldots, 24\}: \quad \dot{\tau}_{g}^{c}=\sum_{s \in A} h_{g s} \dot{\gamma}_{s} ; \quad \dot{\tau}_{g}=\underline{\dot{\bar{\sigma}}}: \underline{\bar{M}}_{g} .
\end{aligned}
$$

The slip rates $\dot{\gamma}_{s}(s \in A)$ are computed by the fixed point method found in Subsection 4.5 . However, $\underline{\bar{d}}$ and $h_{g s}$ are equal to the last converged values computed by Eqs. (32) and (30).

\section{Sub-step 2.4}

- Compute the length $\delta t^{(j)}$ of $I^{\delta}$ over which Schmid's condition is fulfilled. This condition is automatically fulfilled on the systems belonging to the set of the potentially active slip systems $P^{t_{0}}$ over $I^{\delta}$. Indeed the second equation (17) automatically implies Schmid's condition for the potentially active slip systems. For the other systems $\left(\notin P^{t_{0}}\right)$, the following condition must be checked:

$$
\forall g \notin P^{t_{0}}: \quad \tau_{g}^{t_{0}+\delta t^{(j)}} \leqslant \tau_{g}^{c t_{0}+\delta t^{(j)}}
$$

$\tau_{g}^{t_{0}+\delta t^{(j)}}$ and $\tau_{g}^{c t_{0}+\delta t^{(j)}}$ are computed by using the different rates computed in the previous step:

$$
\forall g \notin P^{t_{0}}:\left\{\begin{array}{l}
\tau_{g}^{t_{0}+\delta t^{(j)}}=\bar{M}_{g}: \overline{\bar{\sigma}}^{t_{0}+\delta t^{(j)}}=\tau_{g}^{t_{0}}+\delta t^{(j)} \underline{M}_{g}: \dot{\overline{\bar{\sigma}}}, \\
\tau_{g}^{c t_{0}+\delta t^{(j)}}=\tau_{g}^{c t_{0}}+\delta t^{(j)} h_{g s} \dot{\gamma}_{s} .
\end{array}\right.
$$

The combination of Eqs. (24) and (25) with the first condition $\left(\delta t^{(j)} \leqslant \Delta t\right)$ gives the following condition on $\delta t\left(\delta t^{(j)}\right.$ must, of course, be greater than 0$)$ :

$$
\delta t^{(j)}=\min \left\{\Delta t, \frac{\tau_{g}^{c t_{0}}-\overline{\bar{M}}_{g}: \overline{\bar{\sigma}}^{t_{0}}}{\overline{\bar{M}}_{g}: \underline{\dot{\bar{\sigma}}}-\dot{\tau}_{g}^{c}} ; \forall g \notin P^{t_{0}}\right\} .
$$

If $\left|\delta t^{(j)}-\delta t^{(j-1)}\right|<\varepsilon\left(\varepsilon\right.$ is typically chosen to be equal to $10^{-5}$ ) then $\delta t^{(j)}$ is the correct length $\delta t$ of the sub-increment $I^{\delta}$; go to sub-step 2.5. Otherwise, set $j \leftarrow j+1$ and go to sub-step 2.2 again.

\section{Sub-step 2.5}

- Update the problem variables and go to Section 4.2 again:

$$
\begin{aligned}
& \underline{\sigma}^{t_{0}} \Leftarrow \underline{\sigma}^{t_{0}+\delta t} \quad ; \quad \underline{r}^{t_{0}} \Leftarrow \underline{r}^{t_{0}+\delta t} \quad ; \quad \gamma_{g}^{t_{0}} \Leftarrow \gamma_{g}^{t_{0}+\delta t} \quad ; \quad \tau_{g}^{c t_{0}} \Leftarrow \tau_{g}^{c t_{0}+\delta t}, \\
& \Delta t \Leftarrow \Delta t-\delta t \quad ; \quad t_{0} \Leftarrow t_{0}+\delta t,
\end{aligned}
$$

where

* The rotation $\underline{r}^{t_{0}+\delta t}$ is equal to the last converged value computed as shown in Section 4.5;

* The stress tensor $\underline{\sigma}^{t_{0}+\delta t}$ is equal to $\underline{r}^{t_{0}+\delta t} \underline{\bar{\sigma}}^{t_{0}+\delta t} \underline{r}^{t_{0}+\delta t T}$, where $\underline{\bar{\sigma}}^{t_{0}+\delta t}$ is equal to $\underline{\bar{\sigma}}^{t_{0}}+\delta t \underline{\overline{\bar{\sigma}}}$ and here $\underline{\dot{\bar{\sigma}}}$ is computed by Eq. (23); 
* The accumulated slip of the different slip systems $\gamma_{g}^{t_{0}+\delta t}$ is equal to $\gamma_{g}^{t_{0}+\delta t}=\gamma_{g}^{t_{0}}+\delta t \dot{\gamma}_{g}$, where the slip rate $\dot{\gamma}_{g}$ is equal to the last converged value as explained in Section 4.5 for the systems belonging to the set $A$, and equal to 0 for the other slip systems.

* The critical shear stresses $\tau_{g}^{c t_{0}+\delta t}$ of the different systems are equal to $\tau_{g}^{c t_{0}+\delta t}=\tau_{g}^{c t_{0}}+\delta t \dot{\tau}_{g}^{c}$, where $\dot{\tau}_{g}^{c}$ is computed by Eq. (23).

In this incremental algorithm, for the current sub-increment, $\Delta t$ is the remaining increment of time to reach the end of the step. Therefore, Sections $4.2,4.3$ and 4.4 are repeated until $\Delta t$ equals zero.

\subsection{Computation of the slip rates for a given active set}

The system of Eq. (22) is strongly non linear. Its resolution is based on the iterative fixed point scheme. The application of this method involves the following procedure:

- Initialize: $k=0$ :

- Initialize $\dot{\gamma}_{s}^{(0)}$ to the solution of Eq. (21):

$$
\forall s, g \in A: \quad \dot{\gamma}_{s}^{(0)}=\widetilde{\Psi}_{i(g) i(s)}^{t_{0}-1} \widetilde{Z}_{i(g)}^{t_{0}} .
$$

- Initialize $\underline{r}^{(0)}$ to $\underline{r}^{t_{0}}$.

- Initialize $\tau_{g}^{c(0)}(g \in A)$ to $\tau_{g}^{c t_{0}}$, if the hardening law is stress-based. If it is strain based no initialization is required.

- Set $k=1$.

For $(k>0)$ do the following sub-steps:

\section{Sub-step 2.2.1}

- Compute $\underline{r}^{(k)}$ as follows:

$$
\underline{r}^{(k)}=e^{\delta t^{(j)} \underline{\bar{w}}^{e(k-1)}} \underline{r}^{t_{0}} ; \quad \underline{\bar{w}}^{e(k-1)}=\underline{\bar{w}}^{(k-1)}-\underline{\bar{w}}^{p(k-1)}=\underline{r}^{(k-1) T} \underline{w}^{r^{(k-1)}}-\sum_{g \in P} \dot{\gamma}_{g}^{(k-1)}\left(\bar{M}_{g}\right)_{A} .
$$

This equation is the result of the implicit integration of Eq. (2) over $\left[t_{0}, t_{0}+\delta t^{(j)}\right]$.

- Compute the hardening modulus $h_{g s}^{(k)}$ (for $g, s \in A$ ):

* if the hardening law is stress based, then $h_{g s}^{(k)}$ is equal to $h_{g s}\left(\tau_{g}^{c(k)}\right)$, where $\tau_{g}^{c(k)}$ is defined as follows:

$\tau_{g}^{c(k)}=\tau_{g}^{c t_{0}}+\delta t^{(j)} \sum_{s \in A} h_{g s}^{(k-1)} \dot{\gamma}_{s}^{(k-1)} \quad$ with $\quad h_{g s}^{(k-1)}=h_{g s}\left(\tau_{s}^{c(k-1)}\right)$

* if the hardening law is strain based, then $h_{g s}^{(k)}$ is equal to $h_{g s}\left(\gamma_{g}^{(k-1)}\right)$, where $\gamma_{g}^{(k-1)}$ is defined as follows:

$\gamma_{g}^{(k-1)}=\gamma_{g}^{t_{0}}+\delta t^{(j)} \dot{\gamma}_{g}^{(k-1)}$

- Compute $\underline{\bar{d}}^{(k)}$ :

$$
\underline{\bar{d}}^{(k)}=\underline{r}^{(k) T} \underline{d r}^{(k)} .
$$

- Compute $\widetilde{\Psi}_{i(s) i(g)}^{(k)}$ and $\widetilde{Z}_{i(s)}^{(j)}$ :

$$
\Psi_{i(s) i(g)}^{(k)}=\underline{\bar{M}}_{s}: \underline{\underline{\bar{C}}}: \underline{\bar{M}}_{g}+h_{s g}^{(k)} ; \quad Z_{i(s)}^{(k)}=\underline{\bar{M}}_{s}: \underline{\underline{C}}: \underline{\bar{d}}^{(k)} .
$$

- Compute $\dot{\gamma}_{s}^{(k)}(s \in A)$ :

$$
\left\{\dot{\gamma}^{(k)}\right\}=\left[\widetilde{\Psi}^{(k)}\right]^{-1}\left\{\widetilde{Z}^{(k)}\right\} .
$$

Sub-step 2.2.2 If $\operatorname{Max}\left|\dot{\gamma}_{s}^{(k)}-\dot{\gamma}_{s}^{(k-1)}\right|<\varepsilon$ (for $s \in A$ ) then $\dot{\gamma}_{s}^{(k)}$ is the solution of (22), otherwise set $k \leftarrow k+1$ and go to sub-step 2.2.1. $\varepsilon$ is typically chosen to be equal to $10^{-5} s^{-1}$.

The matrix $\left[\widetilde{\Psi}^{(k)}\right]$ may be singular. Thus its inverse is replaced by its pseudo inverse in the iterations $k$ before convergence. However, when this matrix is singular in the converged iteration, the perturbation technique is used in order to avoid this singularity. The resulting incremental algorithm is conveniently summarized below. 


\section{Set 1: identify the mechanical behavior over the sub-increment $I^{\delta}$}

- Identify the set of the potentially active slip systems $P^{t_{0}}$ at $t_{0}$ by using Eq. (9)

- If the mechanical behavior is elastic, then go to set 2; otherwise go to set 3.

\section{Set 2: Elastic case of the algorithm}

- Use the fixed point method in order to compute the length $\delta t$ of $I^{\delta}$ (steps 1,2 and 3 of Section 4.3.).

- Update the mechanical variables over $I^{\delta}$ (step 4 of Section 4.3.) and go to Set 1.

\section{Set 3: Plastic case of the algorithm}

- Use the combinatorial research procedure in order to identify the set of the active slip systems. If the matrix $\Psi^{t_{0}}$ is singular then use the perturbation technique and go back to Set 2 (elastic case); otherwise go to the following point.

- Use the fixed point method in order to compute the length $\delta t$ of $I^{\delta}$ (step 2 of Section 4.4).

- Use the fixed point method in order to compute the slip rates of the different active slip systems at the end of $I^{\delta}$ (Section 4.5). If the matrix $\Psi$ is singular then use the perturbation technique and go back to Set 2 (elastic case); otherwise go to the following point.

- Update the mechanical variables over $I^{\delta}$ (sub-step 2.5.) and go to Set 1.

\subsection{Use of the perturbation technique}

Thanks to numerous numerical predictions, we noted that the loss of uniqueness ambiguity (i.e., a singular matrix or several solutions given by the combinatorial search procedure) occurred only when the stress state is located on a degenerated side. So, in our case, we considered that the loss of uniqueness ambiguity is the consequence of these degenerations, which are due to the equality between the various critical shear stresses. However, this equality seems to be physically unrealistic because real materials always contain imperfections. For instance, imperfections can result from the presence of a non-zero dislocation density created during the formation of the crystals (Hamelin et al., 2011; Lee et al., 2010; Lemaitre and Chaboche, 1985; Lim et al., 2011). This density may produce a weak but non-zero latent hardening, which may, in turn, generate a small difference between the critical shear stresses. Therefore, we decided to add a minor (so that we can consider it is acceptable to the experimental tolerances), random, positive perturbation to the initial critical shear stresses: each $\tau_{s}^{c 0}$ is replaced by $\tau_{s}^{c 0}\left(1+\alpha_{s} 10^{-3}\right)$ with $\alpha_{s}=\alpha_{s+12}$ chosen randomly in the interval [0,1]. From a practical point of view, after the application of this technique, we noted the total disappearance of these degenerations. Consequently, the perturbation technique appears to be an adequate alternative for the resolution of the loss of uniqueness ambiguity.

\subsection{A few numerical remarks about the integration scheme developed}

- Rem. 1: From our numerical predictions, we observed that a single application of the perturbation technique is sufficient to guarantee the uniqueness of the solution for the subsequent time increments. Also, when the perturbation technique is applied, the matrix $\widetilde{\Psi}$ always becomes invertible for the combinations and time increments. So the use of the pseudoinversion technique, as requested in Section 4.5 is not necessary.

- Rem. 2: The implicit algorithm developed can be transformed easily into an explicit one. Indeed if the value of the slip rates computed by the combinatorial research procedure (21) are used to compute the rate of the different mechanical variables (23) the algorithm becomes explicit. Thus, one may avoid the use of the fixed point method in Section 4.5 to update the slip rates of the different slip systems and the iterative computation of the length $\delta t$ of $I^{\delta}$.

- Rem. 3: The slip rates are slowly evolving variables over $I^{\delta}$. So, the first guess $\dot{\gamma}_{g}^{t_{0}+\delta t^{(j)}}$ is already a good estimation of the final solution. Additionally, the rotation of the crystal lattice over $I^{\delta}$ also evolve slowly in the case of a purely elastic state (Step 2 of the elastic algorithm). Then the iterative computation of $\delta t$ (Sub-step 2.4. of the plastic algorithm) seldom requires more than three iterations.

- Rem. 4: Similarly, th fixed point method used in Subsection 4.5 seldom requires more than three iterations because the lattice rotation and the hardening modulus $h$ are relatively stable and evolve slowly.

- Rem. 5: To improve the computational efficiency, it is suitable to choose the set $P^{t_{0}}$ as a set of active slip systems in the first combination of the combinatorial search procedure (Step 1 of the plastic algorithm). Indeed, barring changes in the deformation path or elastic unloading, it is very probable that such a set $P^{t_{0}}$ is the set of active systems over $I^{\delta}$. Otherwise, if, for any system $g$, the solution $\dot{\gamma}_{g}^{t_{0}}$ (the solution of Eq. (21)) is negative or zero, then this system is inactive and is removed from the list of active slip systems. Hence a reduced system (21) is defined, and solved until all $\dot{\gamma}_{g}^{t_{0}}$ of the systems $g \in A$ become strictly positive.

- Rem. 6: In the application of the fixed point method (Section 4.5), the choice of the trial solution is important to minimize the number of iterations, thereby improving the efficiency of the resolution method. 
- Rem. 7: During the first sub-increments, the stress state is elastic $\left(\bar{\sigma}^{0}=0\right.$ for $\left.t_{0}=0\right)$ until it reaches the first facet of the yield surface, which corresponds to the activation of the first slip system. Then, the stress state moves along the first facet until it activate a second slip system. In this manner, other facets are reached progressively in different sub-increments until arriving at a vertex of the yield surface. Fig. 2 illustrates the sequence of first encountering a facet of the rate independent crystal yield surface and then moving along this facet under a single slip until a second facet (corresponding to another slip system) is reached. This 2-D illustration explains the progression of the algorithm. Note that, during this process, some systems may be activated simultaneously. Generally, at this stage, only one sub-increment (for typical values of strain increments in FE simulations, which are about $0.1-1 \%$ of strain) is required in order to reach the next facet. So, for these sub-increments, the iterative computation of the time sub-increment $\delta t$ is required as $\delta t$ is generally smaller than $\Delta t$. However, the iterative computation of $\delta t$ is only necessary during the first sub-increments, when the stress state is not yet located at a vertex of the yield surface (see next remark).

- Rem. 8: When the stress state at $t_{0}$ is laid on a vertex, $\underline{\bar{C}}: \underline{\bar{d}}$ is generally an outward vector with respect to the yield surface (see Fig. 2). As this yield surface is a convex space, the quantity $\underline{\bar{M}}_{g}: \underline{\overline{\bar{\sigma}}}-\dot{\tau}_{g}^{c}$ is negative for all $g \notin P^{t_{0}}$. This implies that $\delta t$ is equal to $\Delta t$ (see Eqs. (12) and (26)) even in the first iteration (for the computation of $\delta t^{(1)}$ ), which means that the iterative computation of $\delta t$ converges directly to the final value $\Delta t$.

- Rem. 9: The algorithm still works when the different components of the hardening matrix $\mathrm{h}_{\mathrm{sg}}$ become equal to zero. This corresponds to the case of the perfect plasticity. In this case, Eq. (15) becomes:

$$
\forall s \in P^{t_{0}}, \forall t \in I^{\delta}: \quad \dot{\gamma}_{s}^{t} \geqslant 0 ; \quad \dot{\tau}_{s}^{t} \leqslant 0 ; \quad \dot{\gamma}_{s}^{t} \cdot \dot{\tau}_{s}^{t}=0
$$

This reduction of the Eq. (15) leads to an NLCP very similar to the form described in the paper. In this particular case (perfect plasticity), the matrix $\Psi$ of Eq. (33) is written in the following form:

$$
\Psi_{i(s) i(g)}^{(k)}=\underline{\bar{M}}_{s}: \underline{\underline{\bar{C}}}: \underline{\bar{M}}_{g} .
$$

Contrarily, the application of the integration scheme in this particular case becomes easier (in term of number of iterations and CPU time) than the application of the same scheme in the general case (where the components of the hardening matrix $h_{\mathrm{sg}}$ are different from zero). This is due to the reduction of the non linearity level of the NLCP.

\subsection{Comparison with other numerical algorithms}

- Our algorithm takes into account the rotation of the crystal lattice which is not the case of all the proposed algorithms (for example see Miehe and Schröder, 2001; Schröder and Miehe, 1997).

- Unlike the algorithms developed in Anand and Kothari (1996) and Knockaert et al. (2000), our algorithm is fully implicit. Indeed, the combinatorial research procedure is used in order to identify the set of active slip systems. Then, the slip rates are computed by using the fixed point method. This choice allows the value of the rotation (Eq. (29)) and the critical stresses (Eq. (30)) to be considered at the end of the time increment. In comparison, in the algorithms developed in Anand and Kothari (1996) and Knockaert et al. (2000), the slip rates are computed by using the combinatorial research procedure without the fixed point method, which implies that the critical shear stresses are evaluated at the beginning of the time increment. Consequently, the NLCP (19) reduces to an LCP. Even though the use of explicit algorithms allows the problem

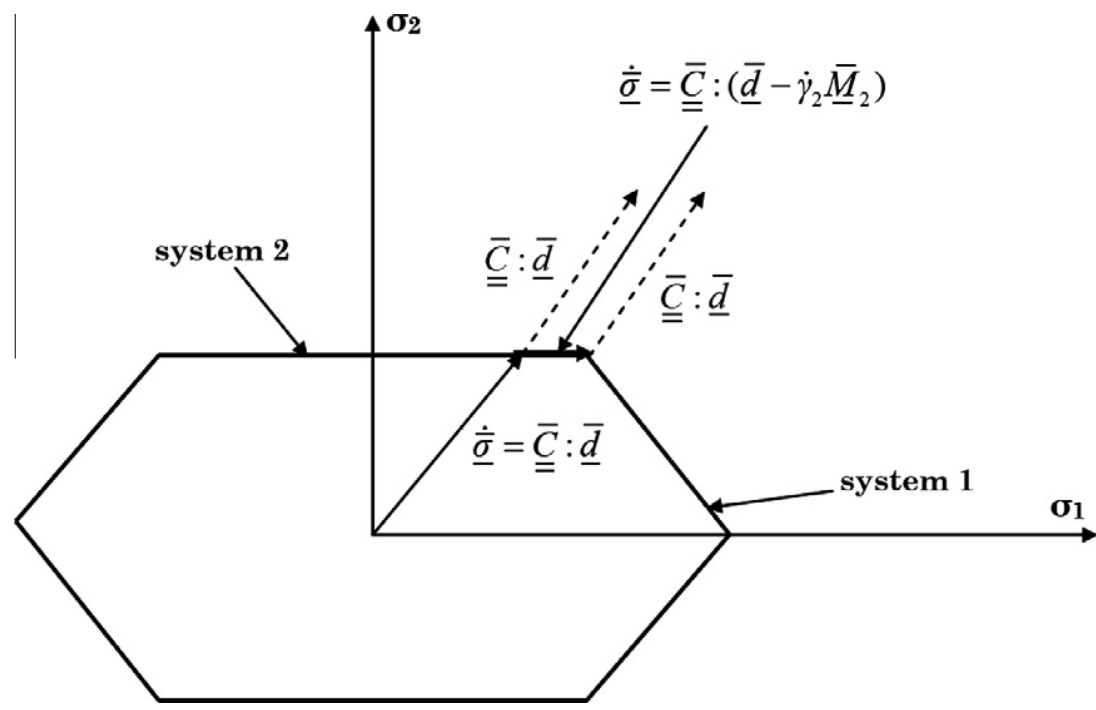

Fig. 2. Evolution of the stress state on a $2-\mathrm{D}$ schematic representation of the single crystal yield surface. 
of the iterative computation of the sub-increment $\delta t$ to be avoided, it is widely known from multiple scientific publications that implicit algorithms are more stable, exhibit good convergence properties and are best suited for larger time increments. Likewise, the implicit procedure proposed in the present paper is quite stable and is valid for any slip system hardening law and for any number of active slip systems.

- The use of the perturbation technique at the beginning of the computation makes it possible to eliminate the indetermination problem linked to the degeneration of the yield surface for the subsequent time increments. In addition, the pseudo inversion technique (e.g., used in Anand and Kothari, 1996; Knockaert et al., 2000), is applied to the different time increments to identify the solution when the matrix $\Psi$ is singular. However, it is not able to choose a solution when the number of solutions is finite (i.e., when the matrix $\Psi$ is non-singular and not positive). Fortunately, the perturbation technique eliminates the various degenerations of the yield surface and always leads to a positive definite matrix $\Psi$.

- In the development of the present algorithm, particular attention was paid to the computation of the length of the subincrement $I^{\delta}$ over which Schmid's law is fulfilled, which is generally not the case in other implicit algorithms.

\section{Numerical results}

In the current subsection, we focus on the accuracy, efficiency and robustness of the numerical schemes proposed. In particular, the algorithm developed is used in order to analyze and study several numerical points:

- Simulation of single crystal behavior: the algorithm developed is used in order to simulate the mechanical behavior of the rate-independent single crystal at a finite strain and finite rotation. The problem of the loss of uniqueness in the active slip systems' set and the corresponding slip rates is thoroughly studied. The perturbation technique is compared with that of pseudo-inversion (Knockaert et al., 2000; Miehe et al., 1999).

- Study of the efficiency and the accuracy of the integration algorithm: the numerical predictions obtained from the present integration algorithm are successfully compared with the numerical predictions presented in Miehe and Schröder (2001). This comparison validates the accuracy of the developed algorithm. The compression test presented in Skalli et al. (1985) is simulated in this section. The numerical predictions in terms of texture evolution are favorably compared with the experimental results.

- Simulation of polycrystal behavior: the overall mechanical behavior of a polycrystal is computed by combining the implicit algorithm with Taylor's homogenization scheme (Van Houtte, 1987; Taylor, 1938a,b). The effect of the number of grains and of the technique used to solve the non-uniqueness ambiguity on the overall response of the polycrystal is analyzed.

- Sensitivity study of the effect of the perturbation technique on the mechanical response of a polycrystal: in the field of single crystal behavior modeling with a rate-independent formulation, an important issue of the different algorithms is to solve the well-known ambiguity of the computation of the slip rates of the active slip systems. Comparisons between different techniques have been carried out by various authors: (Knockaert et al., 2000) compared the pseudo-inversion technique with viscous regularization based on the rate-dependant formulation. In addition (Miehe and Schröder, 2001) compared the pseudo-inversion technique with the diagonal shift method. They concluded that the latter technique is the simplest, most efficient concept. To the authors' knowledge, the study of the effect of these techniques on mechanical behavior at the polycrystal scale has not yet been undertaken in the literature. So, in the present paper a statistical study is carried out in order to study the effect of the perturbation technique on the overall response of the polycrystal. This statistical study points out that the effect of the technique diminishes with the number of grains constituting the polycrystal.

\subsection{Material data}

Except when explicitly stated otherwise, the material studied was Waspaloy, whose parameters were provided in AbdulLatif et al. (1998). The elasticity was assumed to be isotropic with a Young's modulus and a Poisson's ratio taken as (Ben Bettaieb, 2006)

$$
E=215 \mathrm{GPa} ; \quad v=0.3 .
$$

A strain-based hardening law was adopted with the hardening modulus $h_{\alpha \beta}$ defined by the following equation:

$$
h_{\alpha \beta}=Q H_{\alpha \beta} e^{-b\left(\gamma_{\beta}+\gamma_{\beta+12}\right)},
$$

where $\gamma_{\beta}$ is the accumulated slip of the system $\beta$ and $Q$ and $b$ are given as:

$$
Q=256 \mathrm{MPa} ; \quad b=12.6 .
$$

In Eq. (2), $H_{\alpha \beta}$ denotes the components of a square matrix $H$. From experimental observations, Franciosi and Zaoui (1991) suggested a general form of this matrix in the case of FCC materials. This form depends on six interaction coefficients $h_{1}-h_{6}$ :

The numbering of the slip systems presented in the table above is given in Appendix A. The coefficient $h_{1}$ on the diagonal of $H$ describes self-hardening whereas the other terms $\left(H_{\alpha \beta}, \alpha \neq \beta\right.$, having values from $h_{2}$ to $\left.h_{6}\right)$ describe latent hardening, i.e. 
the potential of a slip system to be hardened by the activity of other slip systems. When $h_{1}$ is the sole non-zero coefficient of $H$, the hardening is called diagonal, whereas when all the coefficients are identical, the hardening is called isotropic. The choice of the hardening law described by Eqs. (2) and (3) and Table 2 was prompted by the fact that it includes several hardening forms (i.e., isotropic, diagonal, and anisotropic). Furthermore, the saturation of the hardening is well modeled by this law. Indeed, by increasing slips on the various systems, the behavior with hardening tends toward perfect plastic behavior, which is consistent with the basis of the crystal microstructure.

The six coefficients $h_{1}-h_{6}$ are given in Abdul-Latif et al. (1998) for Waspaloy crystals (Table 3):

To illustrate the non-uniqueness ambiguity and to investigate the influence of the perturbation technique numerically on the response of a single crystal, our simulations were also carried out with isotropic and diagonal hardening:

- Isotropic hardening: $h_{1}=h_{2}=h_{3}=h_{4}=h_{5}=h_{6}=1$.

- Diagonal hardening: $h_{1}=10, h_{2}=h_{3}=h_{4}=h_{5}=h_{6}=0$.

The initial critical resolved shear stress was assumed to be the same for every slip system and equal to 240 MPa (AbdulLatif et al., 1998).

\subsection{Single crystal simulations}

\subsubsection{Loading}

Except when explicitly stated otherwise, the initial conditions of the simulations carried out in the current section are characterized by:

$$
\sigma^{0}=\left[\begin{array}{lll}
0 & 0 & 0 \\
0 & 0 & 0 \\
0 & 0 & 0
\end{array}\right], \quad(s=1, \ldots, 24): \quad \gamma_{s}^{0}=0 \quad \text { and } \quad r^{0}=I d
$$

with Id as the identity second-order tensor. The initial orientation $r^{0}$ holds such that the $\{100\}$ directions of the lattice coincide with the 1-2-3 coordinates.

Therefore, the first sub-increment is purely elastic.

In this paper (except Section 5.2.2), the loading corresponds to a monotonic stretching test characterized by the following constant velocity gradient:

$$
l^{t}=l=\left[\begin{array}{ccc}
0.6 & 0 & 0 \\
0 & -0.3 & 0 \\
0 & 0 & -0.3
\end{array}\right]\left(s^{-1}\right) ; \quad t \in[0,1] ; \quad \Delta t=0.02
$$

The numerical results are expressed as functions of the accumulated strain $e_{11}=t l_{11}$.

Taking into account the initial conditions and the expression of the velocity gradient $l$, we deduce that the first sub-increment $I^{\delta}$ is always purely elastic. At the beginning of the second sub-increment, eight slip systems $\{1,2,4,5,7,9,23,24\}$ are

Table 2

\begin{tabular}{|c|c|c|c|c|c|c|c|c|c|c|c|c|}
\hline System number & 1 & 2 & 3 & 4 & 5 & 6 & 7 & 8 & 9 & 10 & 11 & 12 \\
\hline 1 & $h_{1}$ & $h_{2}$ & $h_{2}$ & $h_{5}$ & $h_{4}$ & $h_{5}$ & $h_{5}$ & $h_{6}$ & $h_{3}$ & $h_{5}$ & $h_{3}$ & $h_{6}$ \\
\hline 2 & & $h_{1}$ & $h_{2}$ & $h_{3}$ & $h_{5}$ & $h_{6}$ & $h_{4}$ & $h_{5}$ & $h_{5}$ & $h_{5}$ & $h_{6}$ & $h_{3}$ \\
\hline 3 & & & $h_{1}$ & $h_{6}$ & $h_{5}$ & $h_{3}$ & $h_{5}$ & $h_{3}$ & $h_{6}$ & $h_{4}$ & $h_{5}$ & $h_{5}$ \\
\hline 4 & & & & $h_{1}$ & $h_{2}$ & $h_{2}$ & $h_{3}$ & $h_{5}$ & $h_{6}$ & $h_{5}$ & $h_{5}$ & $h_{4}$ \\
\hline 5 & & & & & $h_{1}$ & $h_{2}$ & $h_{6}$ & $h_{5}$ & $h_{3}$ & $h_{6}$ & $h_{3}$ & $h_{5}$ \\
\hline 6 & & & & & & $h_{1}$ & $h_{5}$ & $h_{4}$ & $h_{5}$ & $h_{3}$ & $h_{6}$ & $h_{5}$ \\
\hline 7 & & & & & & & $h_{1}$ & $h_{2}$ & $h_{2}$ & $h_{6}$ & $h_{5}$ & $h_{3}$ \\
\hline 8 & Sym & $m c$ & & & & & & $h_{1}$ & $h_{2}$ & $h_{3}$ & $h_{5}$ & $h_{6}$ \\
\hline 9 & & & & & & & & & $h_{1}$ & $h_{5}$ & $h_{4}$ & $h_{5}$ \\
\hline 10 & & & & & & & & & & $h_{1}$ & $h_{2}$ & $h_{2}$ \\
\hline 11 & & & & & & & & & & & $h_{1}$ & $h_{2}$ \\
\hline 12 & & & & & & & & & & & & $h_{1}$ \\
\hline
\end{tabular}

Interaction matrix.

Table 3

Franciosi's matrix coefficients.

\begin{tabular}{llllll}
\hline$h_{1}$ & $h_{2}$ & $h_{3}$ & $h_{4}$ & $h_{5}$ & $h_{6}$ \\
\hline 1 & 0.75 & 0.74 & 1.48 & 1.05 & 3.5 \\
\hline
\end{tabular}


submitted to the same resolved shear stress which is equal to the critical shear stress and holds to be the set of potentially active slip systems (it is the case of the different simulations except Section 5.2.2).

\subsubsection{Accuracy of the algorithm proposed: activation of one slip system}

To assess the performance and the accuracy of the implicit algorithm proposed, a simple test defined by a constant velocity gradient $\bar{l}$ evaluated in the elastically relaxed configuration was simulated. In view of the activation of the first slip system only, $\bar{l}$ was assumed to be equal to $20 . \bar{M}_{1}$. This test is simulated over the time interval $[0, t]$ with $t$ chosen such that $t \cdot \bar{l}_{11}=0.6$. The critical resolved shear stress of the first slip system $\tau_{1}^{c}$ ref , used as a reference, was computed analytically by the integration of the hardening law for the first slip system:

$$
\tau_{1}^{c \text { ref }}=\tau_{1}^{c 0}+\frac{Q}{b} H_{11}\left(1-e^{-b \gamma_{1}^{t}}\right) .
$$

This analytical value was compared with the numerical value $\tau_{1}^{\text {cnum }}$, evaluated using the implicit integration scheme. The relative error between $\tau_{1}^{\text {cnum }}$ and $\tau_{1}^{\text {cref }}$ was less than 0.0002 in the interval $[0, t]$ and its average over this interval was 0.0001 . This example demonstrates the accuracy of the implicit integration algorithm in the case of single slip system activation.

\subsubsection{Study of the non-uniqueness ambiguity}

In this subsection, the behavior law is used in order to analyze the non-uniqueness ambiguity with the different hardening cases (i.e., anisotropic, isotropic, and diagonal).

5.2.3.1. Example of a unique solution: diagonal hardening. At the beginning of the second sub-increment (after the first elastic one), the set of the eigenvalues for $\psi^{t_{0}}$ (see Eq. (18)) is equal to $\{333,329,112,816,112,816,57,688,57,688,2560,2560$, $2560\}$. In this case, eight potentially active slip systems are activated $(\{1,2,4,5,7,9,23,24\})$ and we obtain the equalities $\gamma_{1}^{t}=\gamma_{2}^{t}=\gamma_{4}^{t}=\gamma_{5}^{t}=\gamma_{7}^{t}=\gamma_{9}^{t}=\gamma_{23}^{t}=\gamma_{24}^{t}$ at any time t over [0,1] (Fig. 3(a)). Taking into account these equalities and the equations of the constitutive model, it can be deduced that the orientation remains unchanged $r^{t}=I d$ for $t \in[0,1]$. Consequently, we obtain $\sigma_{22}=\sigma_{33}=-\sigma_{11} / 2$ and $\sigma_{i j}=0$ if $i \neq j$, illustrated in Fig. 3(b). We also compared the response of the single crystal with and without perturbation of the initial critical resolved shear stresses and we noticed that the two responses were almost indistinguishable. The consistency between the two responses is due to the uniqueness property of the solution to the NLCP, which is ensured when the hardening is presumed to be diagonal (see the Appendix D). The perturbation technique makes the choice of one solution among the set of the possible solutions possible. So, when this set contains only one element, the effect of the perturbation technique on the response of the single crystal becomes negligible. In fact, we observed that the perturbation technique modified the path (defined by the order of activation of the slip systems), while the solution (consisting of the set of eight active slip systems) is independent of the perturbation used, as is the solution formed without perturbation.

5.2.3.2. Example of a finite number of solutions: anisotropic hardening. At the beginning of the second sub-increment, the set of the eigenvalues for $\psi^{t_{0}}$ is $\{333,409,110,684,110,684,55,014,54,488,-648,247,67\}$. Thus $\psi^{t_{0}}$ is invertible and its 6 th eigenvalue is negative, so the number of solutions for the NLCP is finite but not necessarily equal to one. The combinatorial search procedure detailed in Section 4.4.3 found three distinct solutions:
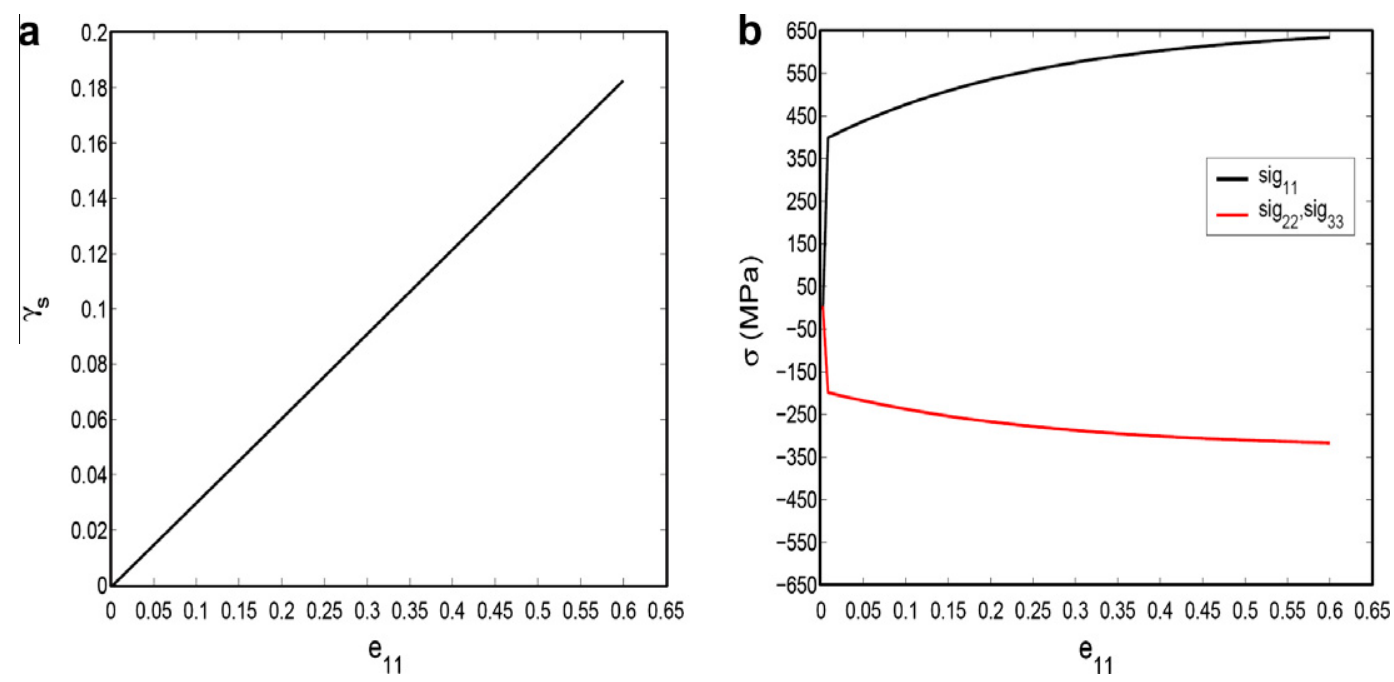

Fig. 3. (a) Accumulated slips $\gamma_{s}$ for the 8 active slip systems versus accumulated strain $e_{11}$ and (b) stress components $\sigma_{i j}$ versus accumulated strain $e_{11}$. 
- The first solution (denoted S1) corresponds to the activation of slip systems 1, 4, 7 and 23, which have identical accumulated slips at every time (Fig. 4(a)).

- The second solution (S2) corresponds to the activation of slip systems 2, 5, 9 and 24 which also have identical accumulated slips at every time. Furthermore, the accumulated slips are equal to the values found in solution $S 1$. This equality is due to the symmetries of the crystallographic structure.

- The third solution (S3) corresponds to the activation of the eight slip systems of solutions $S 1$ and $S 2$ together. Again, they have identical accumulated slips at every time (Fig. 4(a)).

For both $S 1$ and $S 2$, the NLCP has a unique solution during all the remaining sub-increments. In contrast, $S 3$ leads to an NLCP with three more solutions at the beginning of the third sub-increment. These solutions correspond to the activation of slip systems $\{1,4,7,23\},\{2,5,9,24\}$ and $\{1,2,4,5,7,9,23,24\}$. If the last solution is chosen, an identical situation is reproduced at the fourth sub-increment, and so on.

For illustration purposes, the solution $S 3$ was selected at the beginning of each sub-increment for $t \in[0,0.5]$. After $t=0.5$, two strategies can be used:

- Strategy S3-S1, corresponding to the activation of slip systems $1,4,7,23$ for $t>0.5$. These four slip systems have identical accumulated slips at every time (Fig. 4(a)).
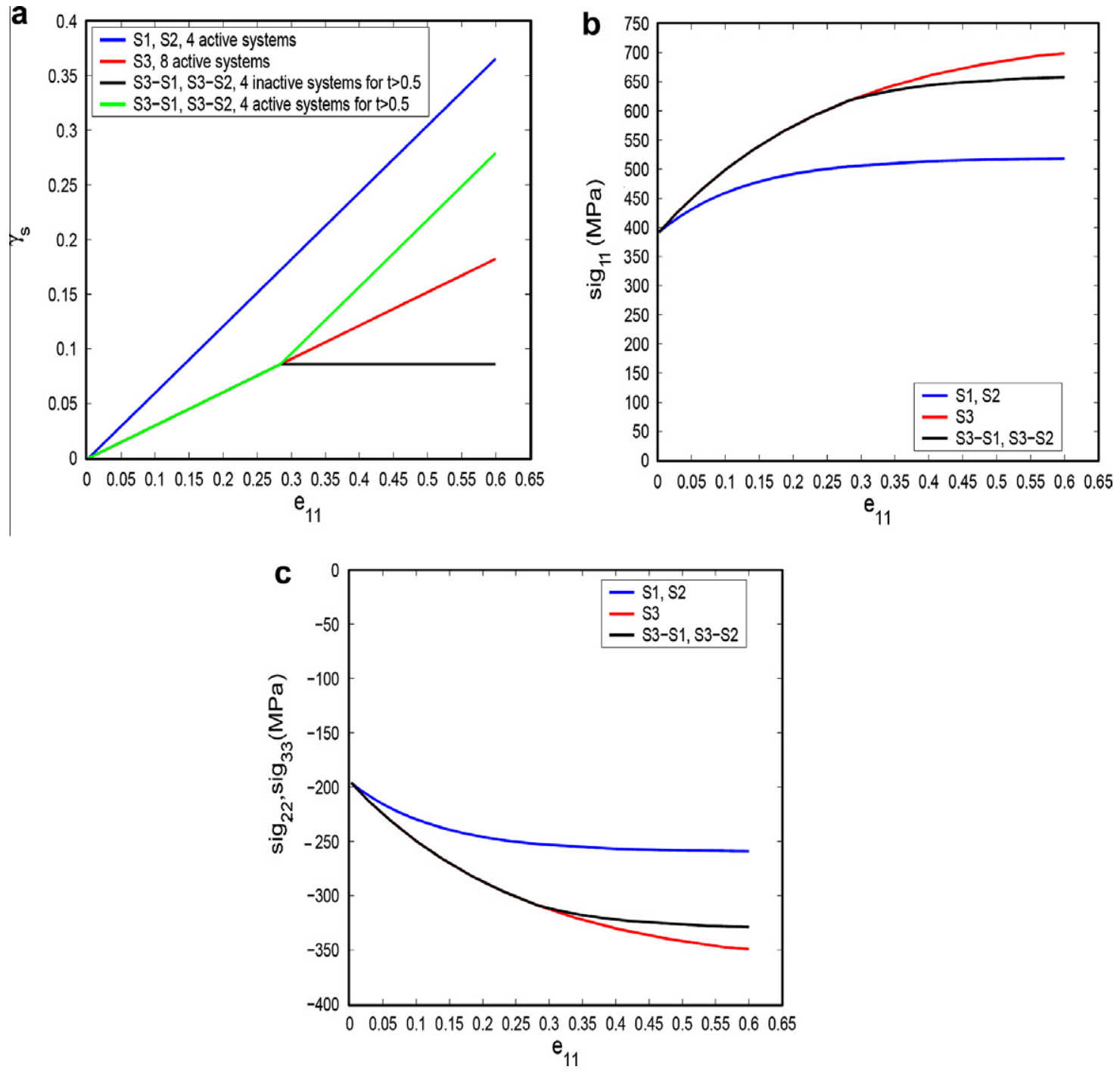

Fig. 4. (a) Accumulated slips $\gamma_{s}$ versus accumulated strain $e_{11}$ for each strategy, (b) $\sigma_{11}$ versus accumulated strain $e_{11}$ and (c) $\sigma_{22}$, $\sigma_{33}$ versus accumulated strain. 
- Strategy S3-S2, corresponding to the activation of slip systems $2,5,9,24$ for $t>0.5$. These four slip systems have also identical accumulated slips at every time with a value equal to the one found in solution S3-S1.

For S3-S1 (or S3-S2) slip systems 2, 5, 9, 24 (or 1, 4, 7, 23, respectively) become inactive over [0.5,1] (constant accumulated slips in Fig. 4(a)).

The evolution of the components of the Cauchy stress tensor, for the five different strategies (S1, S2, S3, S3-S1 and S3-S2) are plotted in Fig. 4(b) and (c). Clearly, the choice of a solution for the NLCP has a marked effect on the response of the single crystal. Taking into account Eqs. (1) and (2), the equality between the accumulated slips of the active systems implies that the rotation tensor remains constant and equal to Id. Thus, the assorted solutions fulfill $\sigma_{22}=\sigma_{33}=-\sigma_{11} / 2$ and $\sigma_{i j}=0$ if $i \neq j$. It appears in Fig. 4 that components of the stress tensor for solutions $S 1$ and $S 2$ (just as $S 3-S 1$ and $S 3-S 2$, respectively) evolve in the same way, although the sets of the corresponding active slip systems are not identical. Furthermore, it can be noted that, after $t=0.5$, solutions $S 3-S 1$ and $S 3-S 2$ behave like $S 1$ and $S 2$, respectively.

Table 4 summarizes the solutions obtained from the NLCP in the case of anisotropic hardening. Symbols $A_{1}, A_{2}$ and $A_{3}$, respectively, stand for the sets of slip systems $\{1,4,7,23\},\{2,5,9,24\}$ and $\{1,2,4,5,7,9,23,24\}$. The application of the perturbation technique at the beginning of the second sub-increment makes it possible to avoid the ambiguity of multiple solutions. Therefore, a unique solution can be found for this sub-increment as well as for the remaining sub-increments. Depending on the value of the perturbation, either solution $S 1$ or $S 2$ can be selected, which shows that these solutions are more stable (and appealing) than solution $S 3$. On the other hand, the application of the pseudo-inverse technique yields solution $S 3$. As previously mentioned, even though $S 1$ and $S 2$ correspond to different sets of active slip systems, they provide the same response of the material in terms of stress components, which proves the superiority of the perturbation technique for this example.

The finite number of solutions in the slip system selection is a numerical artifact resulting from the fact that simulations rely on the "cube" orientation. Such theoretical orientation presents unrealistic, ideal symmetries with regard to the loading axes. In the real material, this situation is never encountered. If a grain would present this orientation, the symmetries with regard to the macroscopic loading axes would be ineffective due to interactions with adjacent grains.

5.2.3.3. Example of an infinite number of solutions: isotropic hardening. In this case, at the beginning of the second sub-increment, the set of the eigenvalues for $\psi^{t_{0}}$ is $\{332,817,110,256,110,256,55128.2,55128.2,0,0,0\}$. The set of the potentially active slip systems at the beginning of the second sub-increment is $\{1,2,4,5,7,9,23,24\}$. Hence, $\psi^{t_{0}}$ is singular and has a rank equal to 5 . So the NLCP has an infinite number of solutions.

One way to overcome the problematic loss of uniqueness is to use the pseudo-inversion technique in order to compute the pseudo-inverse of $\Psi$ as in Anand and Kothari (1996). This technique allows for the selection of one solution, which is denoted by PSI below. Due to the symmetry of the problem and of the crystallographic structure, it was expected that the eight potentially active slip systems would become active simultaneously with identical slip rates at the beginning of the second sub-increment. Consequently, the same situation was reproduced at the beginning of the third sub-increment and the use of the pseudo-inversion was once more necessary; this situation was in fact reproduced for all subsequent sub-increments. As shown in Fig. 5 (a), the eight activated slip systems have identical accumulated slips.

Additionally, the perturbation technique was used to avoid the loss of uniqueness. The application of this technique with two distinct perturbations led to two different responses, which are denoted $P 1$ and $P 2$. For the first (second) response, slip systems 1, 4, 5, 9 and 24 (or 1, 4, 9, 23 and 24, respectively) were activated during deformation (Fig. 5(b) and (c)). The difference between $P 1$ and $P 2$ was due to the permutations of some slip systems as a consequence of structural symmetries of the FCC single crystals: the accumulated slips of systems $1,4,5,9$ and 24 activated in $P 1$ were equal to the accumulated slips of systems $1,4,9,23$ and 24 activated in $P 2$, respectively.

Table 4

Multiplicity of solutions of the NLCP. The numbers in parentheses indicate the number of the selected solution over the total number of possible solutions of the NLCP at the beginning of the current sub-increment.

\begin{tabular}{|c|c|c|c|c|c|}
\hline \multirow{3}{*}{$\begin{array}{l}\mathbf{e}_{\mathbf{1 1}} \\
0 \\
0.002\end{array}$} & \multicolumn{5}{|l|}{ Solutions } \\
\hline & \multicolumn{5}{|c|}{ Purely elastic sub-increment } \\
\hline & $A_{1}(1 / 3)$ & $A_{2}(2 / 3)$ & A3 $(3 / 3)$ & & \\
\hline 0.014 & $A_{1}(1 / 1)$ & $A_{2}(1 / 1)$ & A3 $(3 / 3)$ & & \\
\hline . & $A_{1}(1 / 1)$ & $A_{2}(1 / 1)$ & A3 $(3 / 3)$ & & \\
\hline . & $A_{1}(1 / 1)$ & $A_{2}(1 / 1)$ & A3 $(3 / 3)$ & & \\
\hline . & $A_{1}(1 / 1)$ & $A_{2}(1 / 1)$ & A3 $(3 / 3)$ & & \\
\hline 0.3 & $A_{1}(1 / 1)$ & $A_{2}(1 / 1)$ & $A_{1}(1 / 3)$ & $A_{2}(2 / 3)$ & $A_{3}(3 / 3)$ \\
\hline . & $A_{1}(1 / 1)$ & $A_{2}(1 / 1)$ & $A_{1}(1 / 1)$ & $A_{2}(1 / 1)$ & $A_{3}(3 / 3)$ \\
\hline . & $A_{1}(1 / 1)$ & $A_{2}(1 / 1)$ & $A_{1}(1 / 1)$ & $A_{2}(1 / 1)$ & $A_{3}(3 / 3)$ \\
\hline . & $A_{1}(1 / 1)$ & $A_{2}(1 / 1)$ & $A_{1}(1 / 1)$ & $A_{2}(1 / 1)$ & $A_{3}(3 / 3)$ \\
\hline 0.6 & $A_{1}(1 / 1)$ & $A_{2}(1 / 1)$ & $A_{1}(1 / 1)$ & $A_{2}(1 / 1)$ & $A_{3}(3 / 3)$ \\
\hline Strategy & S1 & S2 & S3 & S3-S1 & S3-S2 \\
\hline
\end{tabular}



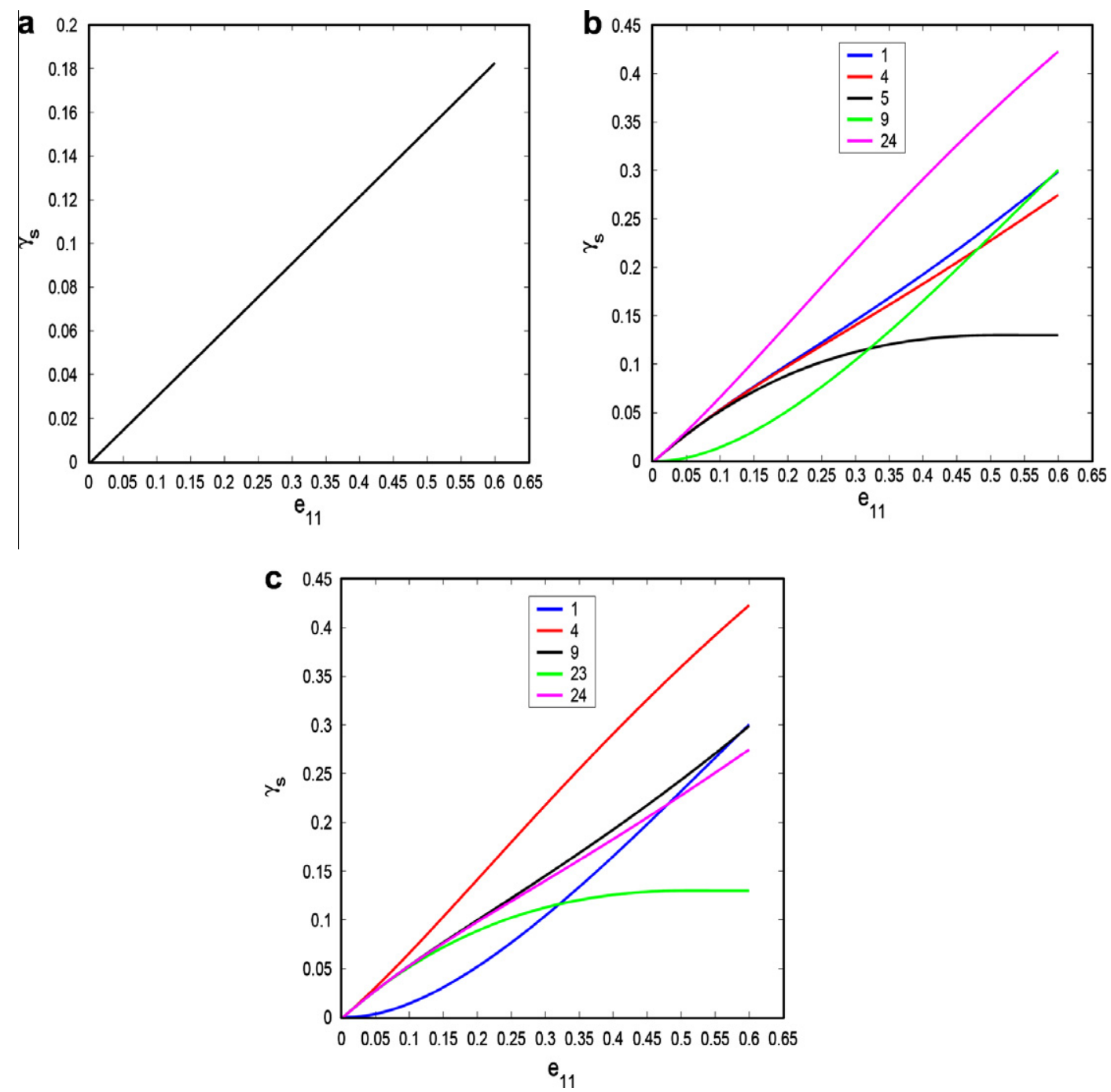

Fig. 5. Accumulated slips $\gamma_{s}$ versus accumulated strain $e_{11}$ : (a) 8 active slip systems of solution PSI, (b) 5 active slip systems of solution P1 and (c) 5 active slip systems of solution P2.

It must be noted that once the perturbation technique is applied (for one of the two distinct perturbations), the solution of the problem becomes unique for the remaining sub-increments; hence it is not necessary to apply it again.

Fig. 6 presents the response of the single crystal in terms of stress components predicted by PSI, P1 and P2; the three solutions are clearly different. Figs. 5 and 6 show that the response of the single crystal is inherently sensitive to the perturbation used.

It may be noted that the three solutions give the same result at the onset of plasticity (at the beginning of the second increment). Referring to the 2D illustration of the perturbation technique given in Fig. 1, the PSIsolution would correspond to the degenerated vertex labeled $A$, while $P 1$ and $P 2$ would correspond to the simple vertices $A^{\prime}$ and $A^{\prime \prime}$, or vice versa. Due to the extremely low value of the perturbation, the stress states on the vertices $A, A^{\prime}$ and $A^{\prime \prime}$ are almost identical at the beginning of the computation. The five activated slip systems obtained using the perturbation technique with any perturbation value always belong to the set of the eight slip systems of the PSI solution. During plastic deformation, as different sets of slip systems are activated for solutions PSI, $P 1$ and $P 2$ (as shown in Fig. 5), the crystal lattice rotation and the critical shear stresses evolve differently for the three solutions (according to Eqs. (2) and (8), respectively). As a result, the stress components diverge for solutions PSI, $P 1$ and $P 2$ (Fig. 6).

It is not easy to determine which of these three solutions is the most acceptable from a physical standpoint. For one, the solution PSI assumes that the eight activated slip systems have exactly the same identical critical shear stress, which is not realistic. On the other hand, solutions $P 1$ and $P 2$ are based on slight but arbitrary differences in the initial critical shear stresses. 

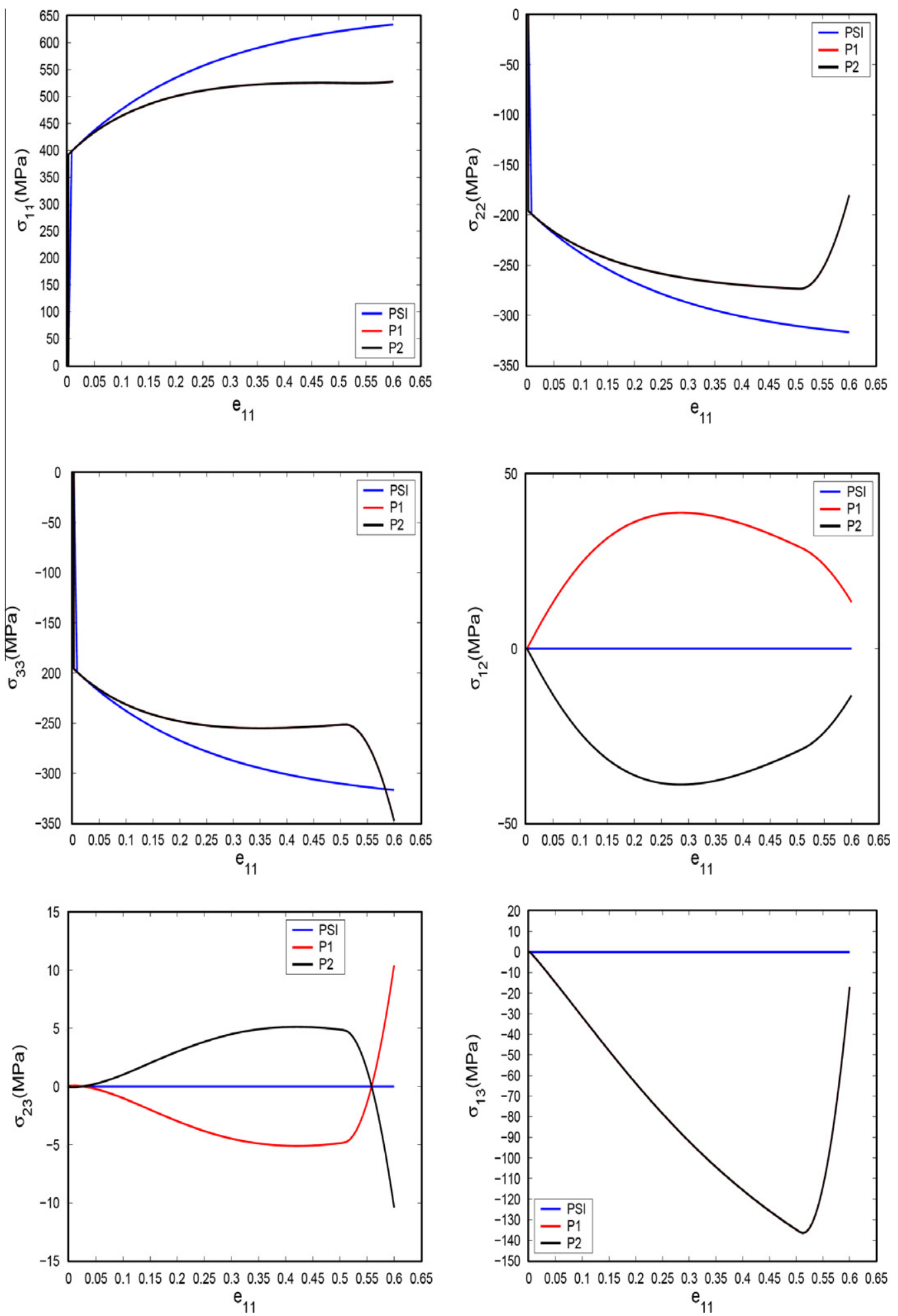

Fig. 6. Stress components $\sigma_{i j}$ versus accumulated strain $e_{11}$.

Furthermore, it is also possible to compare the dissipated plastic work $W_{p}$ for these three solutions (Eq. (43)). It is clear from Fig. 7 that $W_{p}$ is larger in the case of the PSI solution than in the cases of the P1 or P2 solutions, which dissipated the 


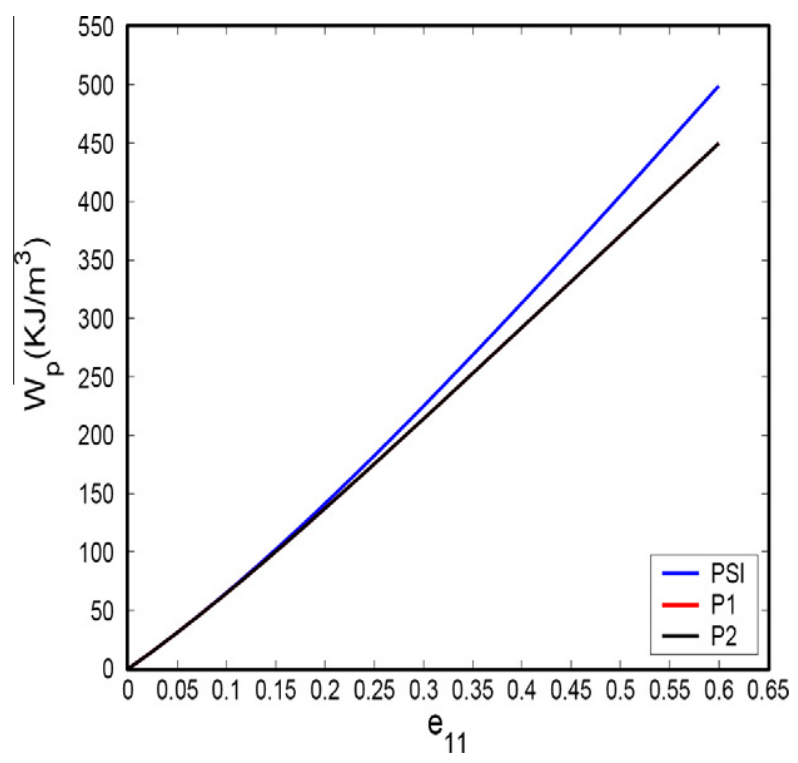

Fig. 7. Plastically dissipated work versus accumulated strain $e_{11}$.

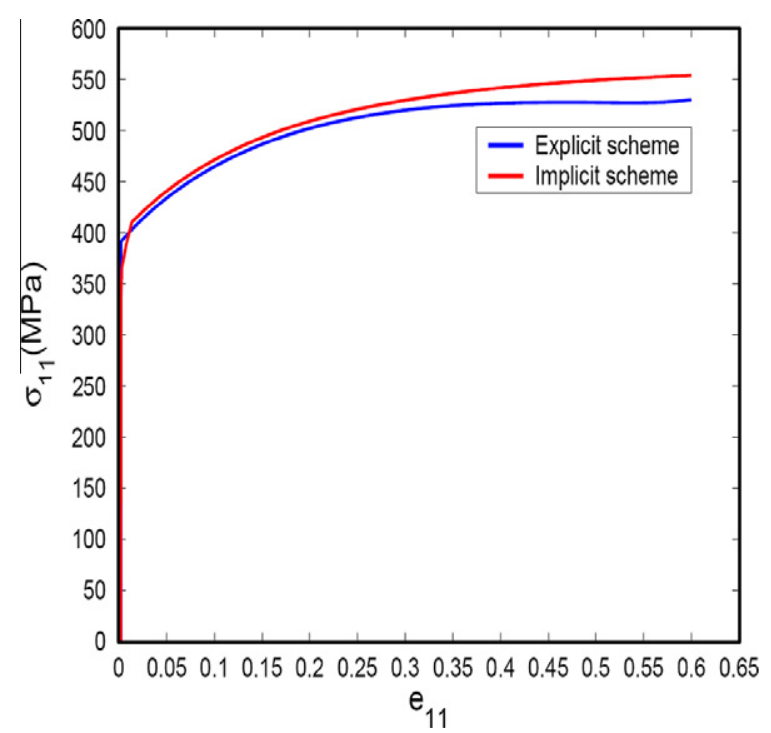

Fig. 8. Comparison between explicit and implicit algorithms.

Table 5

Orientations of the 36 single crystals (Miehe and Schröder, 2001).

\begin{tabular}{|c|c|c|c|c|c|c|c|c|}
\hline No. & $-\varphi_{2}$ & $-\varphi_{1}$ & No. & $-\varphi_{2}$ & $-\varphi_{1}$ & No. & $-\varphi_{2}$ & $-\varphi_{1}$ \\
\hline 1 & 0 & 0 & 13 & 36 & 0 & 25 & 72 & 0 \\
\hline 2 & 0 & 18 & 14 & 36 & 18 & 26 & 72 & 18 \\
\hline 3 & 0 & 36 & 15 & 36 & 36 & 27 & 72 & 36 \\
\hline 4 & 0 & 54 & 16 & 36 & 54 & 28 & 72 & 54 \\
\hline 5 & 0 & 72 & 17 & 36 & 72 & 29 & 72 & 72 \\
\hline 6 & 0 & 90 & 18 & 36 & 90 & 30 & 72 & 90 \\
\hline 7 & 18 & 0 & 19 & 54 & 0 & 31 & 90 & 0 \\
\hline 8 & 18 & 18 & 20 & 54 & 18 & 32 & 90 & 18 \\
\hline 9 & 18 & 36 & 21 & 54 & 36 & 33 & 90 & 36 \\
\hline 10 & 18 & 54 & 22 & 54 & 54 & 34 & 90 & 54 \\
\hline 11 & 18 & 72 & 23 & 54 & 72 & 35 & 90 & 72 \\
\hline 12 & 18 & 90 & 24 & 54 & 90 & 36 & 90 & 90 \\
\hline
\end{tabular}


Table 6

Simple shear stress. Data at $\mathrm{e}_{12}=0.01$ (Miehe and Schröder, 2001).

\begin{tabular}{|c|c|c|c|c|c|}
\hline No. & Active slip systems & $\sigma_{12}$ & No. & Active slip systems & $\sigma_{12}$ \\
\hline 1 & $1,2,4,5,8,19,22,24$ & 2.44949 & 19 & $2,3,6,8,9,23$ & 1.98168 \\
\hline 2 & $1,2,4,5,8,19,22,24$ & 1.98168 & 20 & $2,8,10,11$ & 1.74129 \\
\hline 3 & $1,3,5,6,8,21,22,24$ & 1.54327 & 21 & $4,10,11$ & 1.24311 \\
\hline 4 & $2,3,4,6,9,19,23,24$ & 1.54327 & 22 & $4,6,10$ & 1.24311 \\
\hline 5 & $1,2,4,5,8,19,22,24$ & 1.98168 & 23 & $2,4,6,8$ & 1.74129 \\
\hline 6 & $1,2,4,5,8,19,22,24$ & 2.44949 & 24 & $2,3,6,8,9,23$ & 1.98168 \\
\hline 7 & $1,2,4,5,8,19,22,24$ & 2.32960 & 25 & $2,3,5,6,8,21,23,24$ & 2.32960 \\
\hline 8 & $1,5,8,10$ & 1.87521 & 26 & $2,5,9,11$ & 1.87521 \\
\hline 9 & $1,3,5,6,8,21,22,23$ & 1.46913 & 27 & $1,2,4,5,9,19,22,23$ & 1.46913 \\
\hline 10 & $2,3,4,6,9,19,23,24$ & 1.46913 & 28 & $1,3,4,6,8,19,22,24$ & 1.46913 \\
\hline 11 & $2,4,7,12$ & 1.87521 & 29 & $3,6,8,12$ & 1.87521 \\
\hline 12 & $1,2,4,5,8,19,22,24$ & 2.32960 & 30 & $2,3,5,6,8,21,23,24$ & 2.32960 \\
\hline 13 & $1,2,4,7,8,22$ & 1.98168 & 31 & $2,3,5,6,8,21,23,24$ & 2.44949 \\
\hline 14 & $2,8,10,11$ & 1.74129 & 32 & $2,3,5,6,8,21,23,23$ & 1.98168 \\
\hline 15 & $6,10,11$ & 1.24311 & 33 & $1,2,4,5,9,19,22,23$ & 1.54327 \\
\hline 16 & $4,6,11$ & 1.24311 & 34 & $1,3,4,6,8,19,22,24$ & 1.54327 \\
\hline 17 & $2,4,6,8$ & 1.74129 & 35 & $2,3,5,6,8,21,23,24$ & 1.98168 \\
\hline 18 & $1,2,4,7,8,22$ & 1.98168 & 36 & $2,3,5,6,8,21,23,24$ & 2.44949 \\
\hline
\end{tabular}
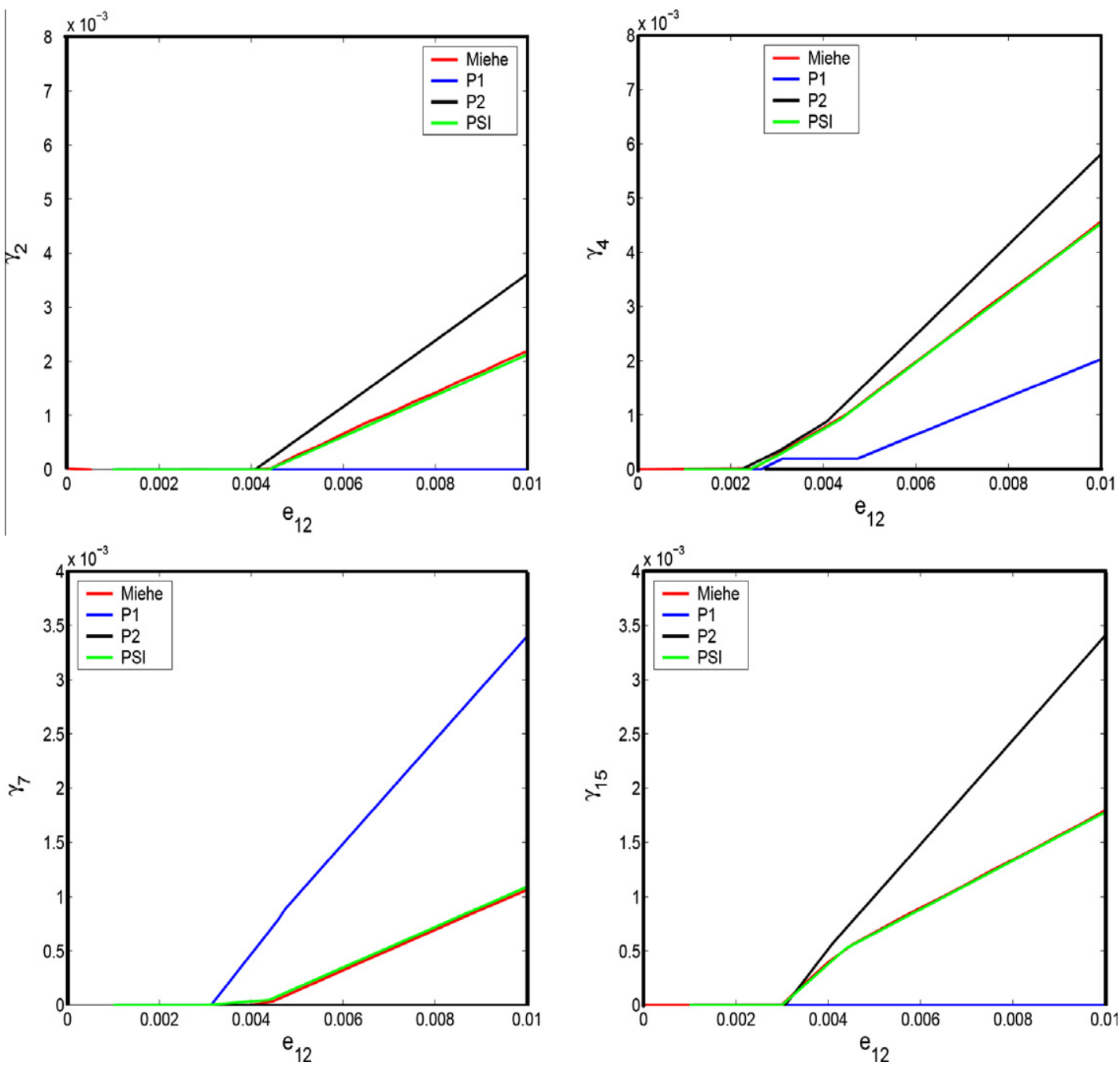

Fig. 9. Accumulated plastic slips versus shear strain $e_{12}$ for the 8 active slip systems $(2,4,7,15,18,21,23,24)$. 

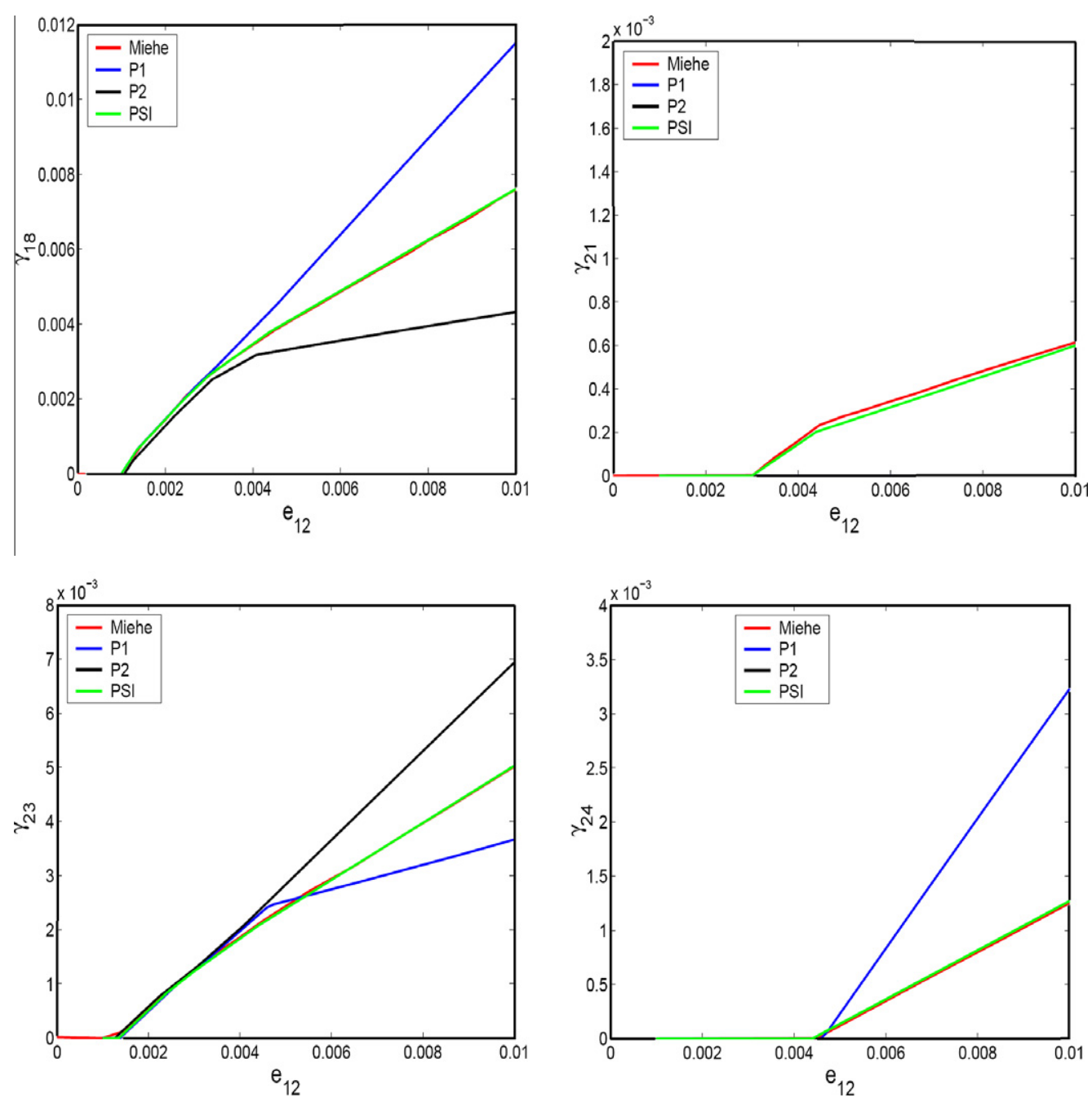

Fig. 9 (continued)

same plastic work. The equality of $W_{p}$ for solutions $P 1$ and $P 2$ can be related to the identical slip rates for these solutions (see Fig. 5(b) and (c))

$$
W_{p}=\int_{0}^{1} \sum_{s=1}^{24}\left(\tau_{s}^{c t} \dot{\gamma}_{s}^{t}\right) d t
$$

\subsubsection{Comparison between explicit and implicit algorithm}

As mentioned in previously, the proposed implicit algorithm can be simplified as an explicit one by avoiding the use of the fixed-point method to update the slip rates of the active slip systems. In order to compare these two integration schemes, isotropic hardening was considered and the same perturbation was applied to the two schemes. Fig. 8 shows that the numerical results obtained by using the implicit algorithm are remarkably similar to those obtained with the explicit algorithm (the time increment $\Delta t$ was $0.02 \mathrm{~s}$ ). This is due to the slow evolution in the slip rates of the different active slip systems. Indeed, the hardening component of the matrix $\Psi$ is small compared to the geometric one. Only the former may change during loading, while the latter is unchanged when the stress state remains located in the same vertex of the yield locus. This means that the change in the slip rates of the different active systems is very slow. This slow evolution implies that few iterations are required in order to update the slip rates for each sub-increment (in practice, 2-3 iterations), which makes the results of the explicit and implicit algorithm so similar. This explanation is consistent with the paper (Knockaert et al., 2000) but with respect to another hardening law. In that paper, the authors demonstrate that there is a slight influence of the length of the time increment on the results of their explicit algorithm.

The CPU time needed for the two algorithms was compared and proved to be nearly equivalent. This is due to the fact that the number of iterations does not exceed 2 or 3 iterations. These iterations are carried out without matrix inversion as 
needed for, e.g. the Newton-Raphson method. This choice limits the time required for each iteration. So the use of the implicit algorithm does not lead to the increase of the CPU time of the simulation.

As demonstrated in Fig. 8, the result of the explicit scheme displays a very low hardening at strains of about 0.55 (this is not a softening). This is due to the non evolution of the slip rates of the active slip systems in this deformation region.

In spite of the similar numerical results and computation time for the two schemes in this case, the development of the implicit algorithm was an ambitious task and is expected to provide more accurate results for particular applications.

\subsubsection{Comparison with Miehe's predictions}

Miehe and Schröder have developed in Miehe and Schröder (2001) an integration algorithm for rate independent single crystals. Their integration algorithm has been used in order to predict the mechanical behavior of several single crystals submitted to simple shear loading. In that paper, the elasticity was assumed to be isotropic. For this purpose, Young's modulus and the Poisson's ratio were equal to $115 \mathrm{GPa}$ and 0.33 respectively. The critical resolved shear stresses were assumed to be constant and equal to $1 \mathrm{MPa}$ for all slip systems (so the hardening effects were neglected). The different crystals were assumed to be stress free in the initial state. A set of 36 different but equally spaced crystal orientations identical to a problem considered in Borja and Wren (1993) was used. The initial orientations of the 36 single crystal are given in Table 5 .

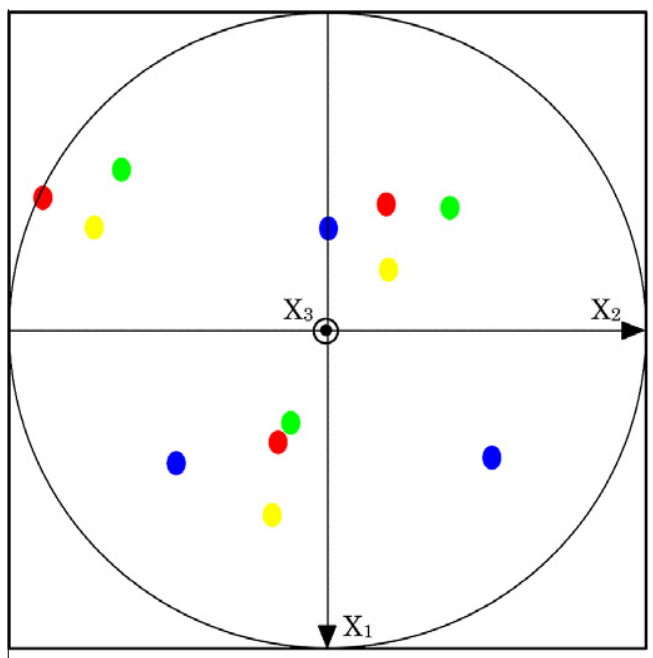

a

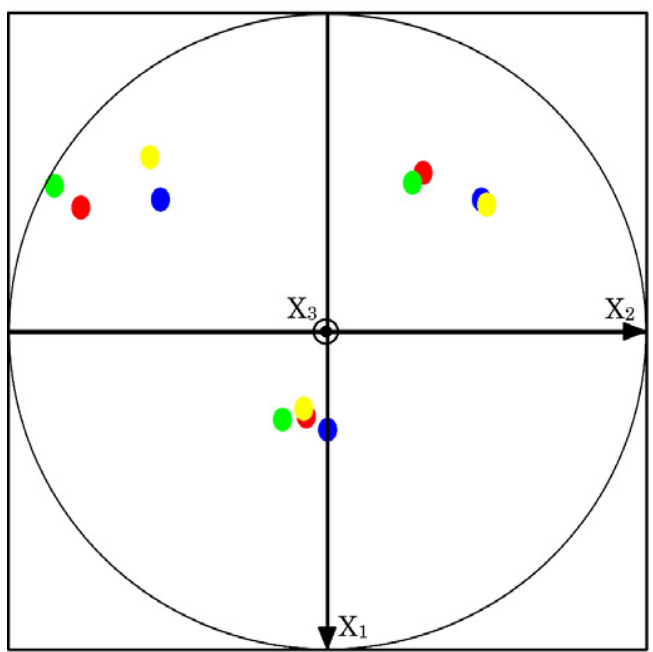

C

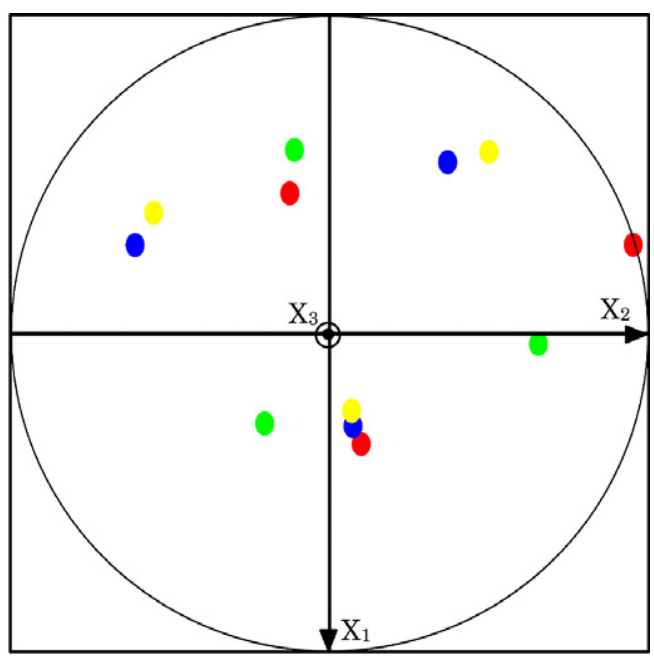

b

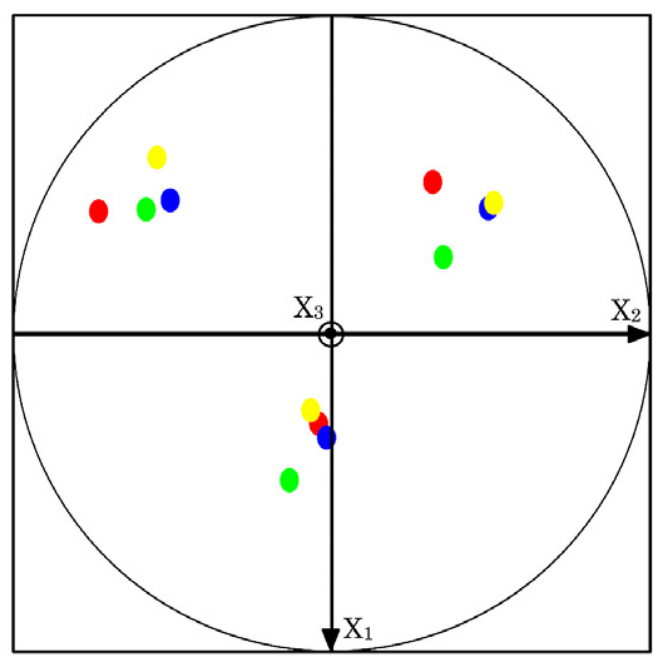

d

Fig. 10. Comparison between our numerical predictions (in green), Driver's predictions (in blue for the FC model and in yellow for the RC model) and experimental results (in red) in term of (100) pole figure: (a) grain 1B, (b) grain 3B, (c) grain 4B, and (d) grain 7B. 
The orientation matrix $\underline{r}$ is related to the angles $\varphi_{1}$ and $\varphi_{2}$ by the following relation:

$$
\underline{r}=\left[\begin{array}{ccc}
\cos \varphi_{1} & \sin \varphi_{1} & 0 \\
-\sin \varphi_{1} & \cos \varphi_{1} & 0 \\
0 & 0 & 1
\end{array}\right]\left[\begin{array}{ccc}
\cos \varphi_{2} & 0 & -\sin \varphi_{2} \\
0 & 1 & 0 \\
\sin \varphi_{2} & 0 & \cos \varphi_{2}
\end{array}\right]
$$

The results of Miehe and Schröder are given in Table 6. By using our numerical algorithm, we obtain exactly the same state of activity of the slip system and the same stress level.

The slips of the active systems of the single crystal number 10 (see Table 4 of about the numbering of the different single crystals) are given in Fig. 9. This figure shows that the predictions of our integration scheme are very close to Miehe's predictions when the pseudo-inversion technique is used. The same stress level than in Borja and Wren (1993) was also obtained. This comparison is a validation of the accuracy of our integration algorithm. The sets of the active slip systems predicted by using the perturbation technique (here two different perturbations $P 1$ and $P 2$ were used) are different from the set determined when the pseudo-inversion technique is used with our integration algorithm and they are different from the set depicted by Miehe and Schröder (2001). This result is consistent with the results observed in Section 5.2.3(c).

\subsubsection{Comparison with Skalli et al. experimental results and predictions}

Skalli et al. (1985) have studied (from numerical and experimental point of views) the mechanical behavior of 99.993\% high purity aluminum sheets submitted to a compression test in the thickness direction. The sheets were obtained at the Voreppe research center of Cegedur Aluminium Pechiney by critical strain annealing. Experimentally, the evolution of the
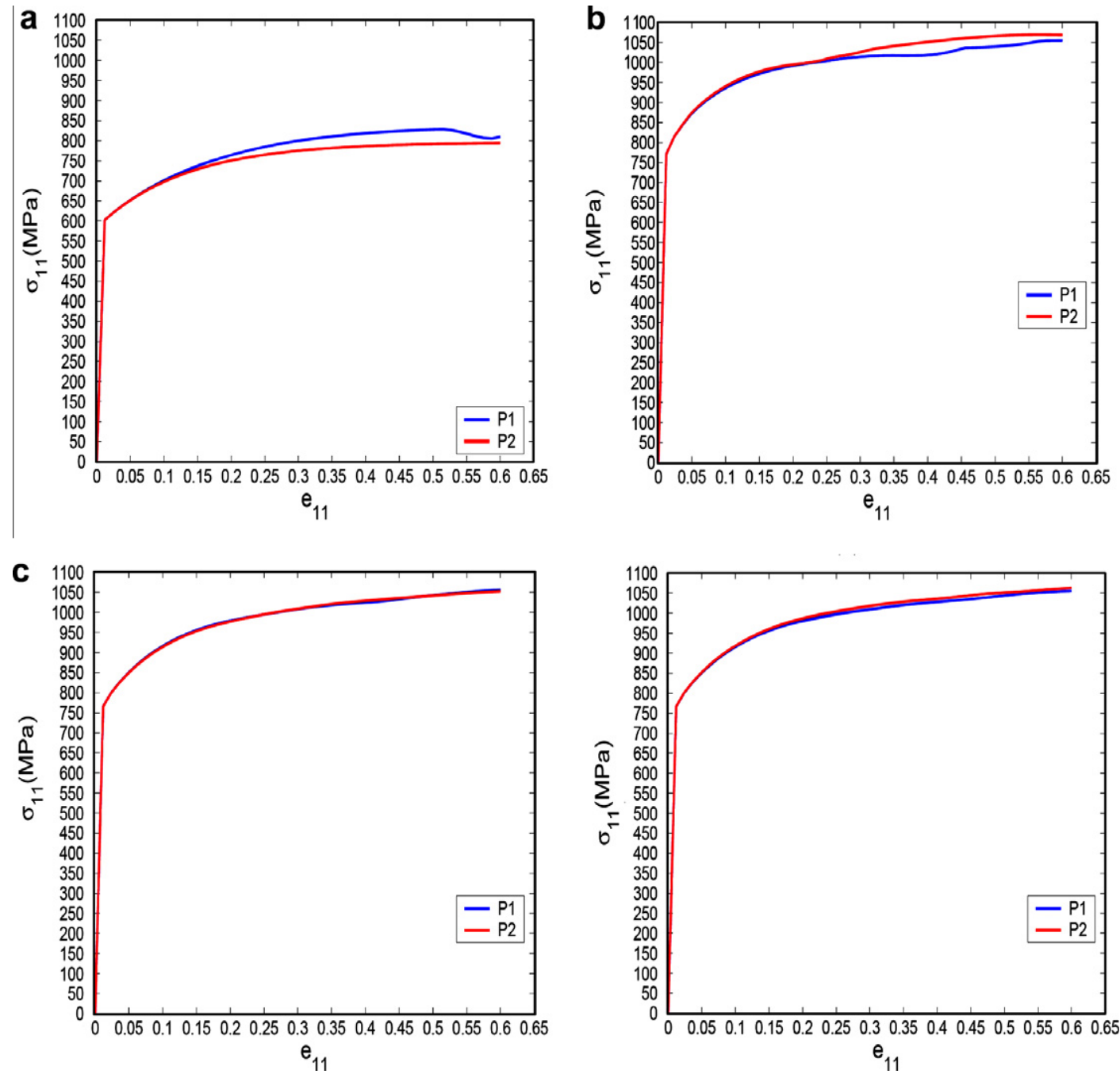

Fig. 11. Effect of the perturbation technique on the overall response of the polycrystal: (a) 1 grain, (b) 10 grains, (c) 100 grains, and (d) 1000 grains. 
different grain orientations were measured on the basis of the grain shapes during compression (Skalli et al., 1985). In their research two sheets were considered: a sheet named A and a sheet named B with thicknesses of 2 and 4 mm respectively. The first sheet contained 8 grains (numbered from $1 \mathrm{~A}$ to $8 \mathrm{~A}$ ). The second sheet contained 11 grains (numbered from $1 \mathrm{~B}$ to 11B). The experimental results for the individual grains are compared with the numerical lattice rotations calculated for different simple grain deformation modes, namely: fully constrained (FC) plane strain compression, and partially constrained plane strain compression in which one or both in-plane shears are not prescribed. In Skalli et al. (1985), the calculations are performed using the classical Bishop-Hill method for FC deformations and the relaxed constraints (RC) method (Honneff and Mecking, 1978; Kocks and Canova, 1981) for partially prescribed grain deformations, together with the Renouard-Wintenberger minimum 2nd order work hypothesis (Renouard and Wintenberger, 1981). The lattice rotations of almost half the grains (1A, 2A, 6A, 8A, 5B, 8B, 11B) were in good quantitative agreement with the RC model. Some grains (4A, 5A and eventually $3 A$ ) rotated according to the $F C$ (Taylor) model along the minimum 2 nd order work path. The rotations of the remaining grains (7A, 1B, 2B, 3B, 4B, 6B, 7B, 9B) were qualitatively close to the rather similar predictions of both models.

Our numerical algorithm was used in order to predict the mechanical behavior of the last set of grains (7A, 1B, 2B, 3B, 4B, 6B, 7B, 9B). These grains were selected from the 19 initial grains because the predictions of Skalli et al. (based on the FC and RC boundary conditions) were close to the experimental results. Our numerical algorithm is used especially to predict the evolution of the lattice rotation. Among the eight selected grains cited above, we have observed that the simulation of the crystal orientation by our numerical algorithm for three grains (1B, 4B, 7B) yielded to accurate results (compared to the experimental measurement of Skalli et al.). For the grains 3B and 9B, the results are farther from the experimental results, while the worst results were obtained for the grains 7A, 2B and 6B. The (100) pole figures corresponding to the lattice rotations at $100 \%$ of compression strain are given in Fig. 10. In these figures, the red, blue, yellow and green dots
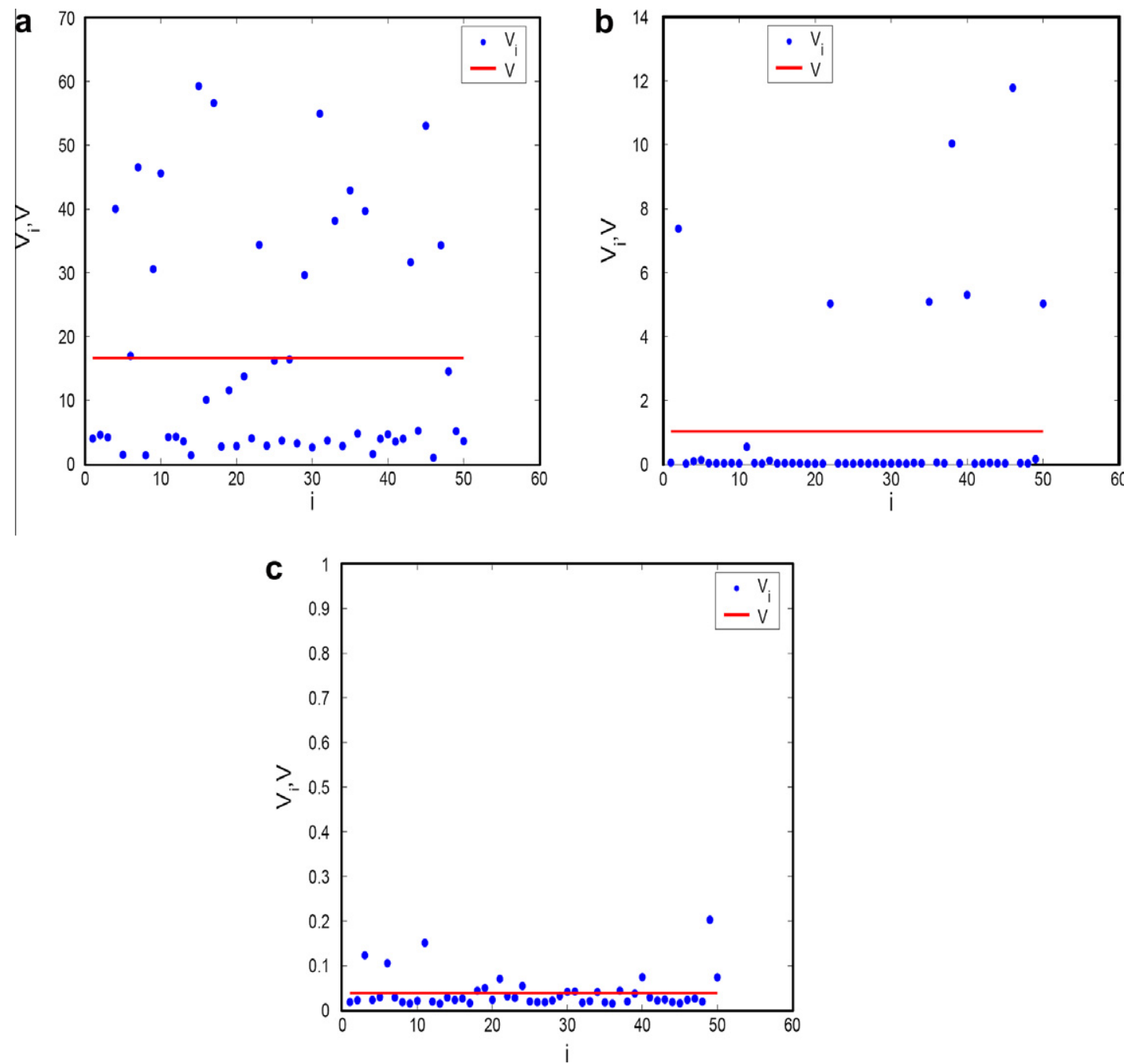

Fig. 12. Normalized variance $V_{i}$ versus test number $i$ : (a) isotropic hardening, (b) anisotropic hardening, and (c) diagonal hardening. 
correspond to the lattice rotation determined experimentally, by using the FC model, by using the RC model and by using our numerical integration algorithm respectively.

So there are some predictions which are very close to the experimental results (closer than the Skalli et al. predictions) and other orientations farther from the experimental lattice rotations. These conclusions are consistent with the conclusions drawn in Skalli et al. (1985).

\subsection{Polycrystal simulations}

\subsubsection{Taylor's model}

In the polycrystal simulations, Taylor's homogenization method (Taylor, 1938a,b, Taylor and Elam, 1923) was used. According to this method, the velocity gradients in all single crystals are the same and are equal to the macroscopic velocity gradient. With regard to the analysis of polycrystalline materials, a quasi-isotropic macroscopic initial state was constructed with a given number of grains that form the microstructure. This initial state of the polycrystalline aggregate was defined by an initial orientation distribution of the grains, which was in turn based on a representation in terms of quaternions as proposed in Bertram et al. (1998). The initial volume fraction of each crystal was identical. The macroscopic velocity gradient $L$ was chosen to be equal to the one used in Subsection 5.2, l, defined by Eq. (41).

\subsubsection{Effect of the perturbation technique on the overall response of the polycrystal}

In the aim of assessing the effect of the perturbation technique on the macroscopic response of the model, the predicted behavior of four polycrystals with 1, 10,100 and 1000 grains, respectively, was analyzed. For each polycrystal, two different perturbations of the critical shear stresses of the different grains were used ( $P 1$ and $P 2$ ). The von Mises equivalent macroscopic stress $\Sigma_{e q}$ is plotted as a function of the 11 components of the macroscopic accumulated strain $E$ ( $E_{11}$ is equal to
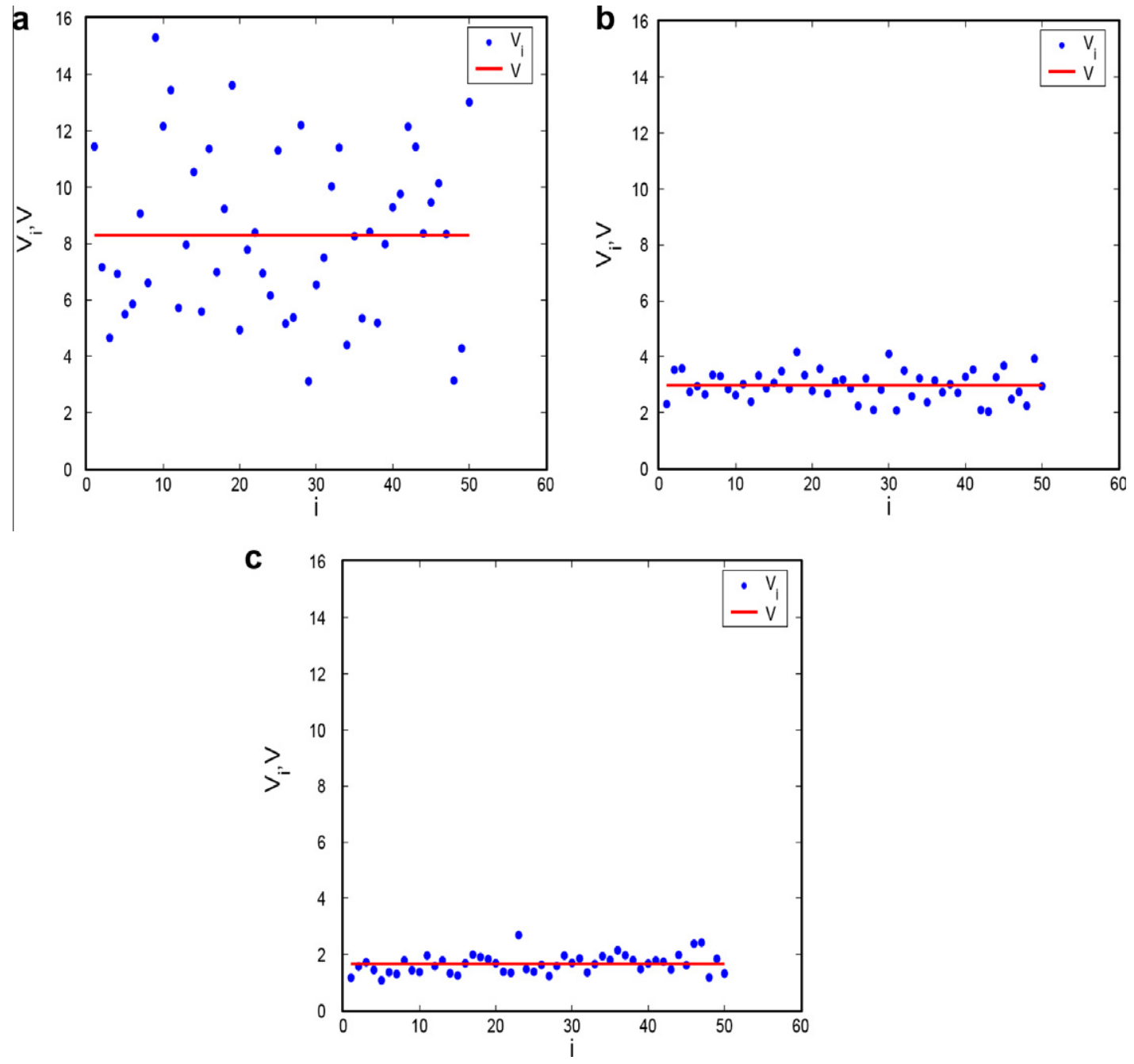

Fig. 13. Normalized variance $V_{i}$ versus test number $i$, for isotropic hardening with different numbers of grains: (a) $m=10$, (b) $m=100$, and (c) $m=1000$. 
t. $\left.L_{11}\right)$ in Fig. 11. It is clear that the effect of the perturbation decreases significantly with an increasing number of grains defining the polycrystal. It appears that the behavior of the polycrystals becomes insensitive to the effect of the perturbation technique when a large number of grains is used. This conclusion is further investigated and quantified in the next subsection. Fig. 11 also shows that the model predicts nearly the same result with 10 or more grains. This observation was also noted by Pourboghrat et al. (2007).

\subsection{Sensitivity study}

In this section, the sensitivity of the mechanical response of an FCC single crystal and a polycrystal to the perturbation of the critical resolved shear stresses is studied. The average response of the polycrystal was computed using Taylor's homogenization method. The material parameters, the initial conditions and the loading of the crystals are set up as in Sections 5.1 and 5.2 .

\subsubsection{Single crystal case}

5.4.1.1. Statistic methodology. To a single crystal, we assign.

- a set $\left\{r^{0 i} ; i=1, \ldots, n\right\}$ of $n$ initial random orientations. Each orientation is used to define one test of the model.

- a set $\left\{\tau^{c 0 j}=\left\{\tau_{s}^{c 0 j}, \tau_{s+12}^{c 0 j}=\tau_{s}^{c 0 j} ; s=1, \ldots, 12\right\} ; j=1, \ldots, p\right\}$ of $p$ initial critical resolved shear stresses, randomly perturbed as indicated in previously.
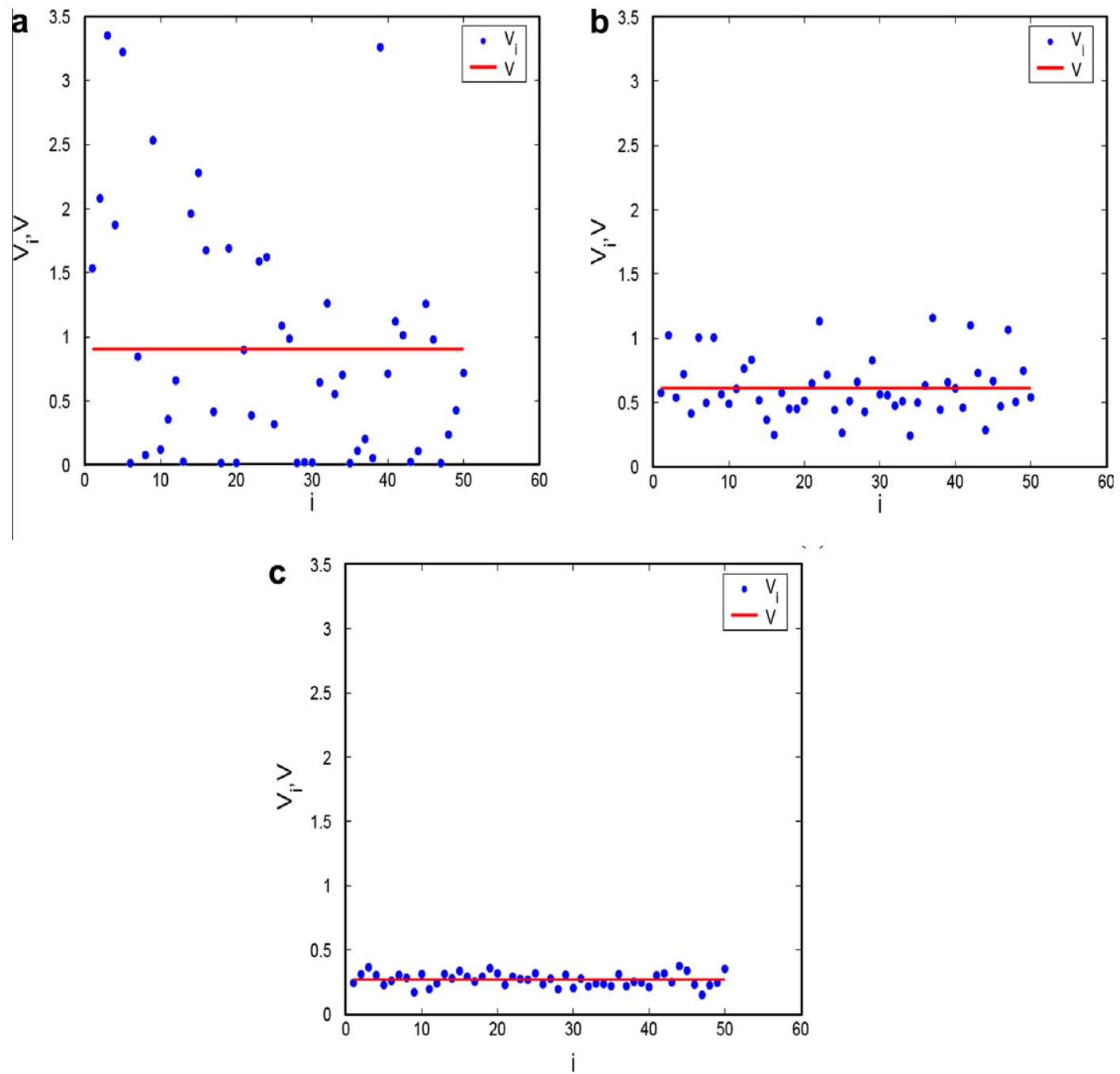

Fig. 14. Normalized variance $V_{i}$ versus test number $i$, for anisotropic hardening with different numbers of grains: (a) $m=10$, (b) $m=100$, and (c) $m=1000$. 
The single crystal is submitted to a stretching test defined by the velocity gradient of Eq. $(41)$ over $[0,1]$. To study the sensitivity of the crystal response to the perturbation of the critical resolved shear stresses, we follow the subsequent statistical analysis:

for $i=1, \ldots, n$

- compute the $i$-th spatial velocity gradient $l^{i}$ applied to the crystal and defined by the rotation $r^{0 i}$ from $l^{i}=r^{0 i T} l r^{0 i}$

- for $j=1, \ldots, p$

- use the integration algorithm to compute the Cauchy stress tensor $\sigma^{i j}$ at $t=1$, corresponding to $l^{i}$ and $\tau^{c 0 j}$

- compute the average tensor $\sigma^{i}=(1 / p) \sum_{j=1}^{p} \sigma^{i j}$ of $\sigma^{i j}$ over the $p$ perturbations and the normalized variance $V_{i}=100^{*}\left(1 / J\left(\sigma^{i}\right) \sqrt{p-1}\right) \sqrt{\sum_{j=1}^{p} J^{2}\left(\sigma^{i j}-\sigma^{i}\right)}$.

- compute the average of the normalized variances over the $n$ tests: $V=(1 / n) \sum_{i=1}^{n} V_{i}$

where $J(\sigma)=\sqrt{(3 / 2) \sigma^{d}: \sigma^{d}}$ defines the von Mises equivalent stress and $\sigma^{d}$ denotes the deviatoric part of $\sigma$.

In practice, to obtain a representative population for the sensitivity study, the values $n=50$ and $p=5$ were selected.

5.4.1.2. Numerical results. Fig. 12 shows the results of this analysis for the different hardening forms presented in Section 5.1 . The solid line in the figure represents $V$, the average of the normalized variance over the 50 tests. The response of the single crystal was highly dependent on the applied perturbation in the case of isotropic hardening (Fig. 12(a)) and less dependent (although the variance is still large) in the case of anisotropic hardening (Fig. 12 (b)). However, sensitivity is negligible when
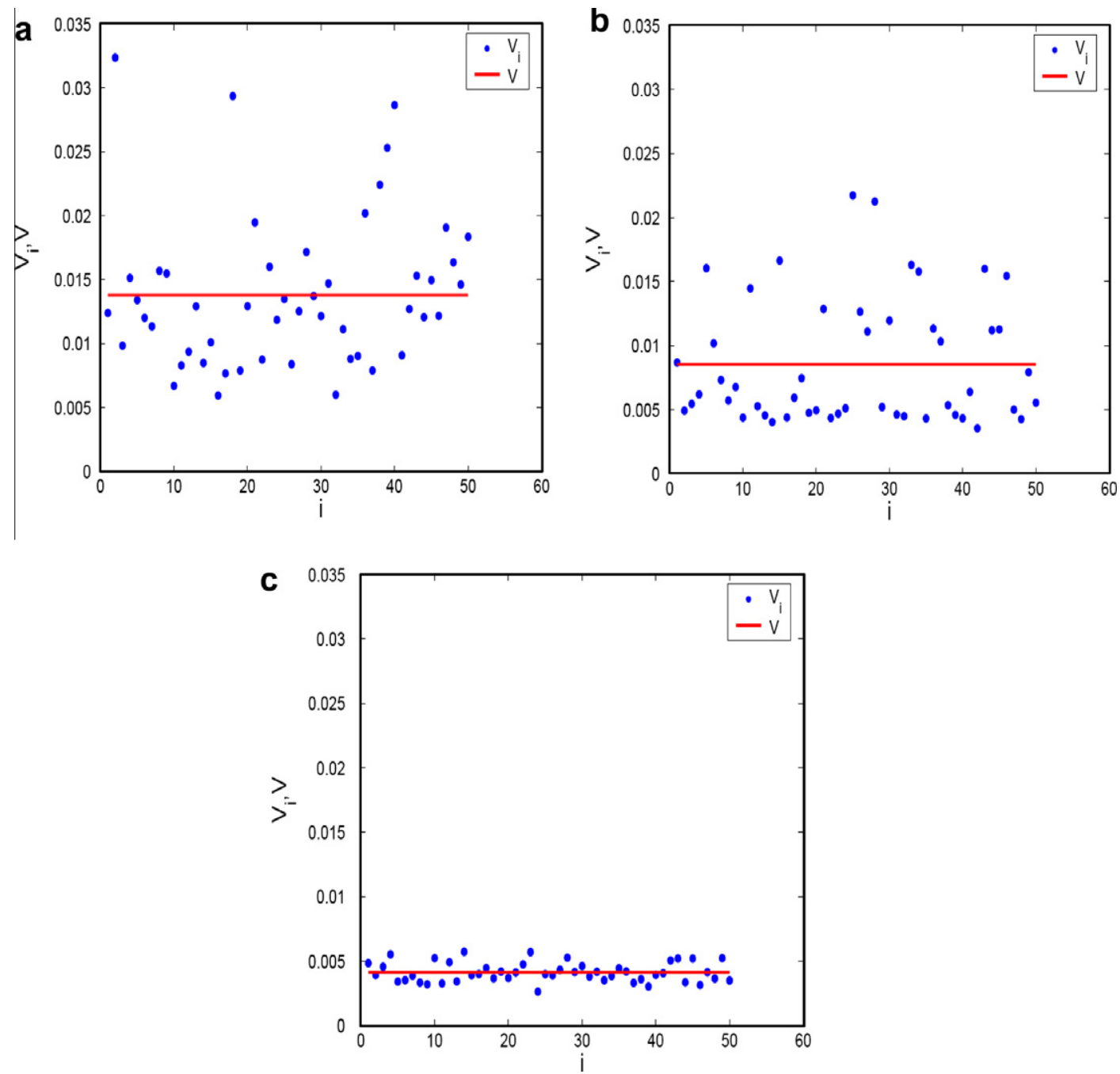

Fig. 15. Normalized variance $V_{i}$ versus test number $i$, for diagonal hardening with different numbers of grains: (a) $m=10$, (b) $m=100$, and (c) $m=1000$. 
the hardening is presumed to be diagonal (Fig. 12(c)). It can be concluded that the evolution of the sensitivity is consistent with the mathematical properties of the $\Psi$ matrix (singularity and positivity). Indeed, in the case of isotropic hardening, the NLCP may have an infinite number of solutions, which favors a wide choice for the set of active slip systems. This wide choice is the origin of the high sensitivity of the single crystal response. In the case of anisotropic hardening, the NLCP has a finite number of solutions. Thus the choice of the solution is not as wide as the preceding case, which causes a lower sensitivity. Finally, the case of diagonal hardening corresponds to a positive definite $\Psi$ matrix, with a unique solution for the NLCP. This involves a low sensitivity of the single crystal response. Interested readers should refer to the Appendix D for further details about the properties of the $\Psi$ matrix for the different hardening forms.

The above numerical results demonstrate that the application of the perturbation technique may lead to significant problems of the model's response sensitivity in the case of single crystals.

\subsubsection{Polycrystal case}

5.4.2.1. Statistical methodology. The polycrystal is made up of $m$ grains whose orientations are defined by the rotation matrices $r^{k}\{k=1, \ldots, m\}$. These rotational matrices are selected randomly in order to model initial isotropic texture for the polycrystal. The material data is identical for all the crystals (as presented in Section 5.2) and the set of randomly perturbed initial critical resolved shear stresses is identical, as in Section 5.1. The polycrystal is submitted to a stretching test defined by an arbitrary rotation of the macroscopic velocity gradient $L$ (defined in Section 5.2.1). To study the sensitivity of the polycrystal to the perturbation technique, we follow the following statistical analysis:
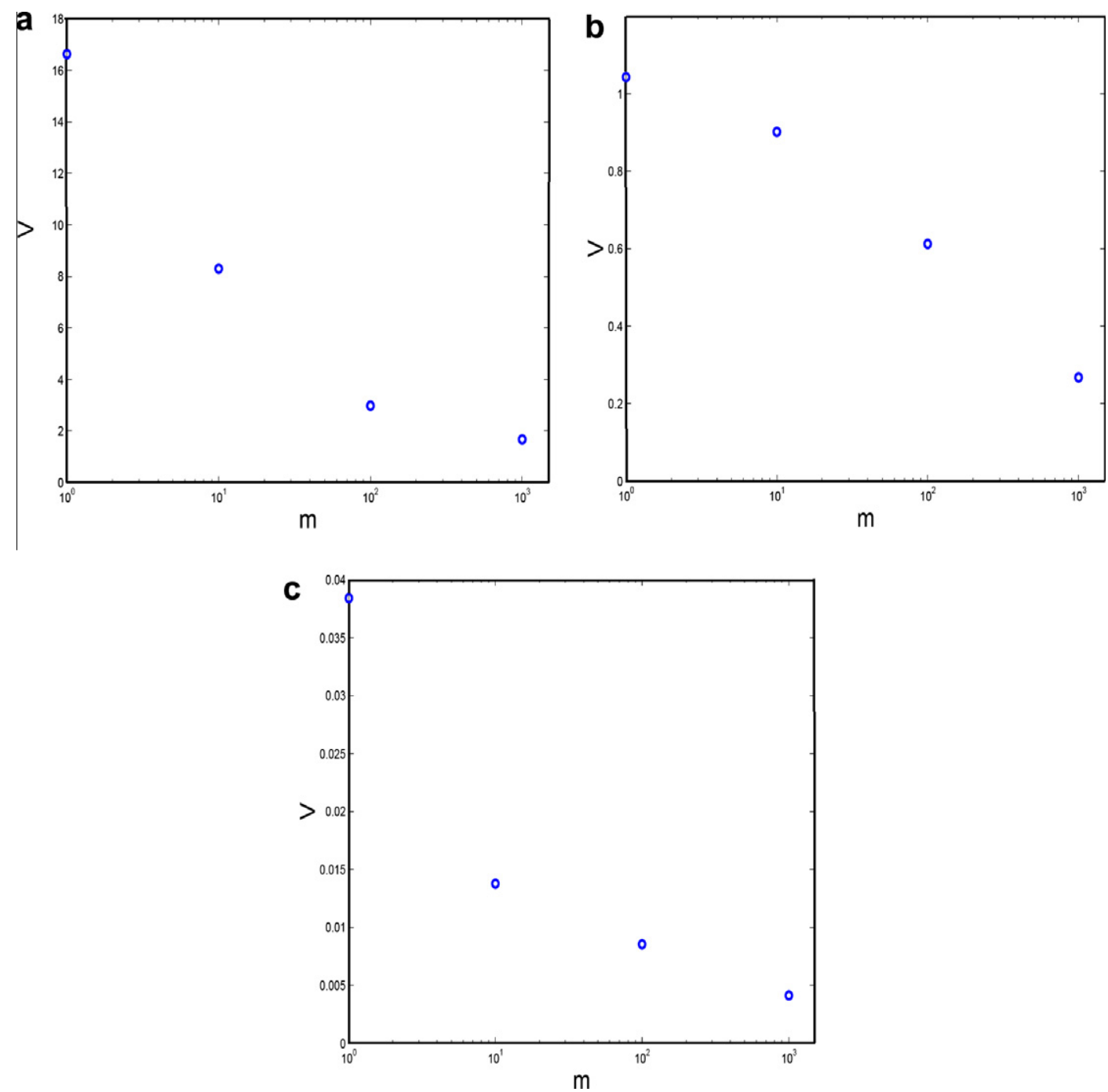

Fig. 16. Average variance $\mathrm{V}$ versus the number of grains $\mathrm{m}$ : (a) isotropic hardening, (b) anisotropic hardening, and (c) diagonal hardening. 


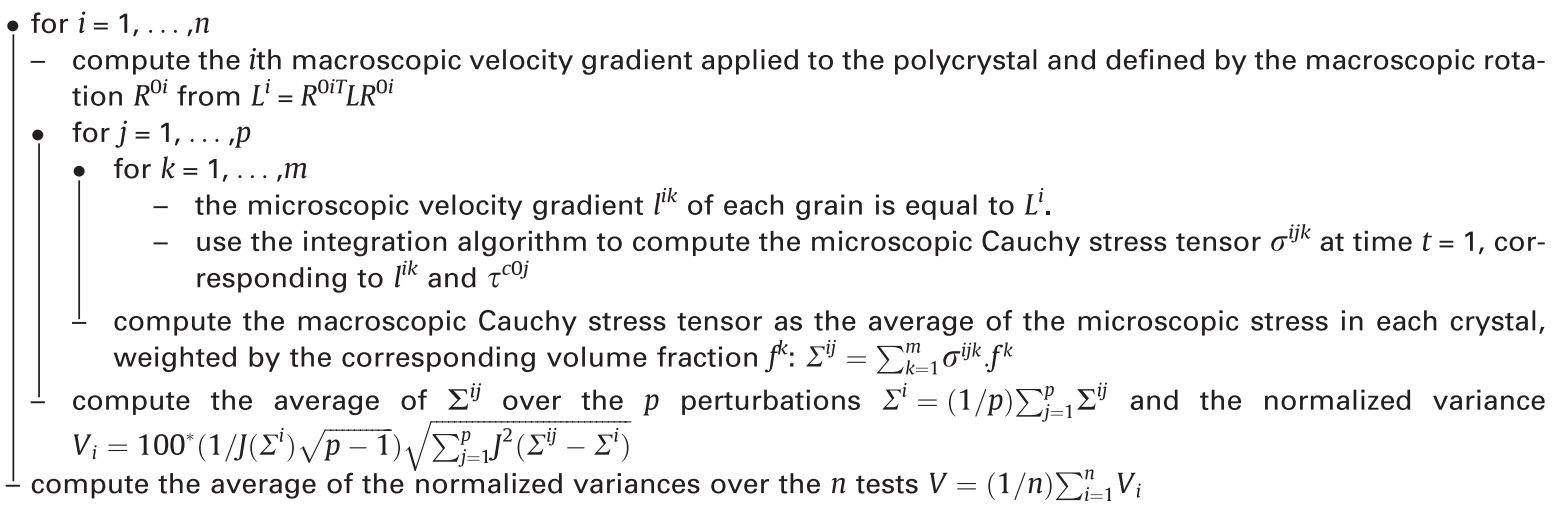

5.4.2.2. Numerical results. In order to numerically capture the effect of the number of grains on the sensitivity of the mechanical response of polycrystals to the perturbation technique, our predictions were performed using several polycrystals: ones with 10, 100 and 1000 grains. The results of this statistical analysis are shown in Figs. 13-15 for the isotropic, anisotropic and diagonal hardening forms, respectively. Clearly the sensitivity of the response to perturbation decreases rapidly as the number of grains increases for all hardening forms. In addition, as previously observed for single crystals, the variance is greatly influenced by the hardening form. The variance is lowest for diagonal hardening while the worst case is observed under isotropic hardening. Fig. 16 presents the evolution of the average of the normalized variances $V$ versus the number of grains; the values obtained in Section 5.4 .1 for single crystals are also plotted. From this figure, it appears that the predicted polycrystalline responses are not significantly sensitive to the perturbation technique when the number of grains is sufficiently large.

\section{Conclusions}

In this paper, a new implicit integration algorithm for modeling the response of FCC single crystals, in the case of rateindependent plasticity and large strain field is proposed. This algorithm is both efficient and accurate (it allows the integration the non linear set of equations issue from the single crystal model without any assumption or approximation). Initially designed for FCC crystal structures, it is general and flexible enough to be extended to other crystal structures such as BCC or HCP and to anisotropic elasticity, and it can be applied to any hardening law. This algorithm can also be transformed easily into an explicit one. In addition, the well-known ambiguity due to the loss of uniqueness is resolved with the perturbation technique. Studying the topology of the yield locus proves that the main cause of this ambiguity is the equality of the initial critical shear stresses. As a result, aside from introducing several rate-dependent approaches, which allow a unique solution to be selected, and the pseudo-inversion technique, the perturbation of the initial critical shear stresses is another way to overcome the ambiguity and to obtain a unique solution. This perturbation makes it possible to account for possible defects or dislocation history of the real materials.

The analysis of the simulation results for the single crystals and the polycrystals yields the following conclusions:

(1) The NLCP derived from the consistency condition may have one solution, a finite number of solutions or an infinite number of solutions. In the literature, the case of an infinite number of solutions has been widely studied, while the case with a finite number of solutions has not been treated enough. This paper gives additional information for this situation.

(2) The type of hardening matrix has a significant influence on the material response due to the activation of different slip systems.

(3) The difference between the simulation results obtained from the explicit algorithm and the implicit one is small. However, it must be noted that the case of simple, monotonic loading was investigated in this study (corresponding to a slow evolution in the slip rates). It is expected that for more realistic, complex loadings this difference would be larger.

(4) The numerical algorithm developed in this paper permits to reproduce correctly the experimental results in some cases and gives less accurate results in other cases.

(5) The statistical study points out that the mechanical response of the single crystal may be highly sensitive to the perturbation technique despite the low value of these perturbations (especially in the case of isotropic hardening). On the contrary, variance due to the application of the perturbation technique is averaged out for polycrystals, provided that the number of grains is large enough. 
Table A.1

The numbering of the slip systems of an FCC single crystal according to BS convention (Boukadia and Sidoroff, 1988).

\begin{tabular}{|c|c|c|c|c|c|c|c|c|c|c|c|c|}
\hline s & 1 & 2 & 3 & 4 & 5 & 6 & 7 & 8 & 9 & 10 & 11 & 12 \\
\hline$\sqrt{3} \overline{\vec{n}}_{s}$ & $\left\{\begin{array}{l}1 \\
1 \\
1\end{array}\right\}$ & $\left\{\begin{array}{l}1 \\
1 \\
1\end{array}\right\}$ & $\left\{\begin{array}{l}1 \\
1 \\
1\end{array}\right\}$ & $\left\{\begin{array}{c}1 \\
1 \\
-1\end{array}\right\}$ & $\left\{\begin{array}{c}1 \\
1 \\
-1\end{array}\right\}$ & $\left\{\begin{array}{c}1 \\
1 \\
-1\end{array}\right\}$ & $\left\{\begin{array}{c}1 \\
-1 \\
1\end{array}\right\}$ & $\left\{\begin{array}{c}1 \\
-1 \\
1\end{array}\right\}$ & $\left\{\begin{array}{c}1 \\
-1 \\
1\end{array}\right\}$ & $\left\{\begin{array}{c}-1 \\
1 \\
1\end{array}\right\}$ & $\left\{\begin{array}{c}-1 \\
1 \\
1\end{array}\right\}$ & $\left\{\begin{array}{c}-1 \\
1 \\
1\end{array}\right\}$ \\
\hline$\sqrt{2} \overline{\vec{m}}_{s}$ & $\left\{\begin{array}{c}1 \\
-1 \\
0\end{array}\right\}$ & $\left.\begin{array}{c}1 \\
0 \\
-1\end{array}\right\}$ & $\left\{\begin{array}{c}0 \\
1 \\
-1\end{array}\right\}$ & $\left\{\begin{array}{l}1 \\
0 \\
1\end{array}\right\}$ & $\left\{\begin{array}{c}1 \\
-1 \\
0\end{array}\right\}$ & $\left\{\begin{array}{l}0 \\
1 \\
1\end{array}\right\}$ & $\left\{\begin{array}{c}1 \\
0 \\
-1\end{array}\right\}$ & $\left\{\begin{array}{l}0 \\
1 \\
1\end{array}\right\}$ & $\left\{\begin{array}{l}1 \\
1 \\
0\end{array}\right\}$ & $\left\{\begin{array}{c}0 \\
1 \\
-1\end{array}\right\}$ & $\left\{\begin{array}{l}1 \\
1 \\
0\end{array}\right\}$ & $\left\{\begin{array}{l}1 \\
0 \\
1\end{array}\right\}$ \\
\hline
\end{tabular}

\section{Acknowledgments}

M. Ben Bettaieb and L. Duchêne would like to acknowledge the Interuniversity Attraction Poles Program - Belgian State Belgian Science Policy (Contract P6/24). The Belgian Fund for Scientific Research FRS-FNRS is also acknowledged.

\section{Appendix A}

For an FCC single crystal, one considers four slip planes and three directions of slip in each plane, hence a total of 12 slip systems. Each slip system is characterized by orthonormal vectors $\left(\overrightarrow{\vec{m}}_{s}, \overrightarrow{\vec{n}}_{s}\right) . \overrightarrow{\vec{m}}_{s}$ is the unit vector parallel to the slip lines and $\overrightarrow{\vec{n}}_{s}$ is the unit vector normal to the slip plane. Both vectors are assumed to be constant directions in the intermediate configuration and to be of unit length, therefore the following relations hold:

$$
\forall s=1, \ldots, 12: \quad\left|\overrightarrow{\vec{m}}_{s}\right|=\left|\overrightarrow{\vec{n}}_{s}\right|=1 ; \quad \overrightarrow{\vec{m}}_{s} \cdot \overrightarrow{\vec{n}}_{s}=0 .
$$

The numbering of $\overrightarrow{\vec{m}}_{s}$ and $\overrightarrow{\vec{n}}_{s}$, measured in the isoclinic configuration, is enumerated in Table A.1. As already mentioned above and in order to simplify the numerical study of the problem, each physical slip system $s$ is broken up into two oriented slip systems. These oriented slip systems are characterized by the subsequent orientation tensors:

$$
\underline{M}_{s}=\left\{\begin{array}{l}
\overrightarrow{\vec{m}}_{s} \otimes \overrightarrow{\vec{n}}_{s} \quad \forall s=1, \ldots, 12, \\
-\overrightarrow{\vec{m}}_{s-12} \otimes \overrightarrow{\vec{n}}_{s-12} \quad \forall s=13, \ldots, 24 .
\end{array}\right.
$$

\section{Appendix B}

We quote here the most important hardening laws used in the case of single crystal plasticity. The first law is stress based (i.e. the coefficients $h_{\mathrm{j}}$ depend directly on $\tau_{j}^{c}$ ). However the others are strain based (i.e. the coefficients $h_{\mathrm{j}}$ depend directly on $\gamma_{j}$.

\begin{tabular}{ll}
\hline Ref. & Hardening law \\
\hline Anand and Kothari (1996) and & $h_{i j}=h_{j}\left[q+(1-q) \delta_{i j}\right] ; \quad h_{j}=h_{0}\left(1-\frac{\tau_{j}^{c}}{\tau_{s a t}}\right)^{a}$ \\
Knockaert et al. (2000) & $h_{0}, q, \tau_{s a t}$ and $a$ are material parameters \\
& $h_{i j} h(A)\left[q+(1-q) \delta_{i j}\right] ; h(A)=h_{0}+\left(h_{\infty}-h_{0}\right)\left[1-\operatorname{sech}^{2}\left(\left(h_{0}-h_{\infty}\right) A / \xi\right)\right]$ \\
& $A$ is the sum of the accumulated plastic slip on all slip systems $h_{0}, h_{\infty}, q$ and $\xi$ \\
Miehe et al. (1999) & are material parameters \\
& $h_{i j}=h(A)\left[q+(1-q) \delta_{i j}\right] ; h(A)=h_{0}+\left(h_{\infty}-h_{0}\right)[1-\exp (-\xi A)]$ \\
& $A$ is the sum of the accumulated plastic slip on all slip systems $h_{0}, h_{\infty}, q$, and \\
Chang and Asaro (1981) & $\tau_{s a t}$ are material parameters \\
& $h_{i j}=h(A)\left[q+(1-q) \delta_{i j}\right] ; h(A)=h_{0} \sec h^{2}\left(\frac{h_{0} A}{\tau_{s a t}-\tau_{0}}\right)$ \\
& $A$ is the sum of the accumulated plastic slip on all slip systems $\tau_{s a t}, \tau_{0}, q$ and $h_{0}$ \\
Ling et al. (2005) and Miehe and & are material parameters \\
Schröder (2001) & $h_{i j}=Q H_{i j}\left(e^{-b\left(\gamma_{j}+\gamma_{j+12}\right)}\right)$ \\
& $H$ is an interaction matrix, $Q$ and $b$ are material parameters \\
Abdul-Latif et al. (1998) and Ben &
\end{tabular}

\section{Appendix C}

The fixed point method is a method of computing fixed points of iterated functions. It is used to solve non linear equation in the form: 


$$
x=f(x) .
$$

Regarding the dependence between the different variables in our paper, we think that the fixed point method is more appropriate to solve our set of equations than other iterative methods (like the Newton-Raphson method for example). So we have decided to choose it.

Practically, this method allows to solve Eq. (C.1) iteratively. After giving an initial solution (also named trial solution) to this problem noted $x_{0}$, the iterative procedure may be written as:

$$
x_{n+1}=f\left(x_{n}\right) ; \quad n=0,1,2, \ldots
$$

This iterative procedure is repeated until verifying that:

$$
\left|x_{i+1}-x_{i}\right|<\varepsilon
$$

where $\varepsilon$ is the required precision. In this case, $x_{i+1}$ can be considered as an approximate solution of Eq. (C.1).

More generally, the function $f$ can be defined on any metric space with values in that same space. So the fixed point method can be applied to solve a non-linear system of equations like in the case of the present paper.

Like the totality of the iterative methods (e.g. the Newton-Raphson method), the convergence of the fixed point method is not always guaranteed and it is very difficult to know, a priori, the number of iterations required to achieve an approximation of the solution at a given precision. The choice of the trial solution is very important to determine a solution to Eq. (C.1) with the minimum number of iterations. In our case, the choice of this trial solution is optimized in order to increase the efficiency of the iterative procedure. We observed that the iterative procedure converges always to a solution which is unique in 2 or 3 iterations.

\section{Appendix D}

It is helpful for the above discussions to briefly discuss the mathematical properties of $\psi$ for the cases of the particular hardening forms used in this paper.

\section{Notation}

A matrix is called semi-positive if it is singular and all of its eigenvalues are positive or null.

The $\Psi$ matrix is broken down into two distinct parts: a geometric component $\Psi^{\text {geom }}$ and a hardening component $\Psi^{\text {hard }}$.

$$
\forall g, s \in P: \quad \Psi_{i(s) i(g)}^{g e o m}=\underline{\bar{M}}_{s}: \underline{\underline{C}}: \underline{\bar{M}}_{g} ; \quad \Psi_{i(s) i(g)}^{\text {hard }}=h_{s g} .
$$

(1) Isotropic hardeningIn this case, $\Psi$ is defined by:

$$
\forall g, q \in P: \Psi_{i(g) i(q)}=\underline{\bar{M}}_{g}: \underline{\underline{C}}: \underline{\bar{M}}_{q}+Q H_{1} \sum_{j=1}^{12}\left(e^{-b\left(\gamma_{j}+\gamma_{j+12}\right)}\right) .
$$

If the stress state lies on a simple $i$-side of the yield locus, $\Psi$ is positive definite since it is the sum of a positive definite matrix (the geometric part) and a semi-positive matrix (the hardening part). If the stress state is situated on a degenerated $i$-side the matrix $\Psi$ may be singular or positive definite. So in the latter case, we cannot conclude a priori on the properties of $\Psi$. The definition of simple and degenerated i-side is given in Subsection 3.2.

\section{(2) Anisotropic hardening}

In this case again, we cannot conclude on the properties of $\Psi$. However, multiple numerical predictions of ours demonstrate that this matrix is always regular but not necessarily positive definite.

\section{(3) Diagonal hardening}

In this case, $\Psi$ is defined by:

$$
\forall g, q \in P: \Psi_{i(g) i(q)}=\underline{\bar{M}}_{g}: \underline{\bar{C}}: \underline{\bar{M}}_{q}+Q H_{1} e^{-b\left(\gamma_{g}+\gamma_{g+12}\right)} \delta_{g q}
$$

The matrix $\mathrm{QH}_{1} e^{-b\left(\gamma_{\mathrm{g}}+\gamma_{g+12}\right)}$ Id is positive definite and the matrix $[\underline{\bar{M}}]_{P}: \overline{\bar{C}}:[\underline{\bar{M}}]_{P}$ is positive definite if the $i$-side is simple, or semi-positive if the $i$-side is degenerated. This implies that $\Psi$ is always positive definite.

\section{References}

Abdul-Latif, A., Dingli, J.P., Saanouni, K., 1998. Modeling of complex cyclic inelasticity in heterogeneous polycrystalline microstructure. Mech. Mater. 30, 287-305.

Anand, L., Kothari, M., 1996. A computational procedure for rate-independent crystal plasticity. J. Mech. Phys. Solids 44, $525-558$.

Arul Kumar, M., Mahesh, S., Parameswaran, V., 2010. A 'stack' model of rate-independent polycrystals. Int. J. Plast..

Asaro, R.J., 1983. Crystal plasticity. J. Appl. Mech.-Trans. ASME 50, 921-934.

Asaro, R.J., Needleman, A., 1985. Texture development and strain hardening in rate dependent polycrystals. Acta Metall. $33,923-953$.

Ben Bettaieb, M., 2006. Modélisation numérique du comportement de matériaux polycristallins par homogénéisation périodique. Université de la Méditerranée, Marseille, France. 
Bertram, A., Böhlke, T., Kraska, M., 1998. Texture development of aluminum polycrystals under finite plastic deformations. In: Bruhns, O.T., Stein, E. (Eds.), Micro- and Macrostructural Aspects of Thermoplasticity, pp. 127-136.

Bishop, J.F.W., Hill, R., 1951a. A theoretical derivation of the plastic properties of a polycrystalline face-centered metal. Philos. Mag. $42,1298-1307$.

Bishop, J.F.W., Hill, R., 1951b. A Theory of the plastic distortion of a polycrystalline aggregate under combined stresses. Philos. Mag. $42,414-427$.

Boukadia, J., 1988. Contribution à l'étude de la viscoplasticité anisotrope en grandes déformations: le cas du monocristal. Ecole Centrale de Lyon, Lyon, France.

Boukadia, J., Sidoroff, F., 1988. Simple shear and torsion of a perfectly plastic single crystal in finite transformation. Arch. Mech. 40, 497-513.

Borja, R.I., Wren, J.R., 1993. Discrete micromechanics of elastoplastic crystals. Int. J. Numer. Methods Eng. 36, 3815-3840.

Chang, Y.W., Asaro, R.J., 1981. An experimental study of shear localization in aluminium-copper single crystals. Acta Metall. 29, $241-257$.

Chenaoui, A., 1992. Contribution à l'étude du comportement du monocristal en grandes déformations plastique. Ecole Centrale de Lyon, Lyon, France.

Chenaoui, A., Sidoroff, F., Hihi, A., 2000. The texture evolution of a planar polycrystal. J. Mech. Phys. Solids 48, 2559-2584.

Chitra, A., Subrahmanyam, P.V., 1987. Remarks on a nonlinear complementarity-problem. J. Optimiz. Theory Appl. 53, $297-302$.

Cottle, R.W., 1966. Programs with positively bounded Jacobians. SIAM J. Appl. Math. 14, 147-158.

Débordes, O., Bettaieb, M., S., B., Dogui, A., 2005. About two questions on micro-macro modeling and simulation in large strain plasticity. In: Plasticity’05, Kauai, USA.

Dogui, A., 1989. Plasticité anisotrope en grandes déformations. Université Claude Bernard, Lyon 1, Lyon, France.

Driver, J.H., Skalli, A., Wintenberger, M., 1984. A theoretical and experimental study of the plastic deformation of f.c.c. crystals in plane strain compression. Philos. Mag. A, 505-524.

Elbououni, S., 2002. Simulation numérique d'anisotropie induite dans les matériaux polycristallins selon une approche micro-macro. Université de la Méditerranée, Marseille, France.

Elbououni, S., Bourgeois, S., Debordes, O., Dogui, A., 2003. Simulation by periodic homogenization of the behavior of a polycrystalline material in large elastoplastic transformations. J. Phys. Iv 105, 123-130.

Franciosi, P., Zaoui, A., 1991. Crystal hardening and the issue of uniqueness. Int. J. Plast. 7, 295-311.

Fortunier, R., Driver, J.H., 1987. A continuous constraints model for large strain grain deformations. Acta Metall., $509-517$.

Gambin, W., 1991. Plasticity of crystals with interacting slip systems. Eng. Trans. 39, 303-324.

Gambin, W., 1992. Refined analysis of elastic-plastic crystals. Int. J. Solids Struct. 29, 2013-2021.

Golub, G.H., Van Loan, C.F., 1983. Matrix Computations. The Johns Hopkins University Press, Baltimore, MD.

Hamelin, C.J., Diak, B.J., Pilkey, A.K., 2011. Multiscale modelling of the induced plastic anisotropy in bcc metals. Int. J. Plast. $27,1185-1202$.

Honneff, H., Mecking, H., 1978. In: Gottstein, G., Lücke, K. (Eds.), Textures of Materials. Springer, Berlin, p. 265.

Hill, R., Rice, J.R., 1972. Constitutive analysis of elastic-plastic crystals at arbitrary strain. J. Mech. Phys. Solids 20, 401-413.

Karamardian, S., 1969a. The nonlinear complementarity problem with applications I. J. Optimiz. Theory Appl. 4, 87-98.

Karamardian, S., 1969b. The nonlinear complementarity problem with applications II. J. Optimiz. Theory Appl. 4, 167-181.

Kanjarla, A.K., Van Houtte, P., Delannay, L., 2010. Assessment of plastic heterogeneity in grain interaction models using crystal plasticity finite element method. Int. J. Plast. 26, 1220-1233.

Knockaert, R., Chastel, Y., Massoni, E., 2000. Rate-independent crystalline and polycrystalline plasticity, application to FCC materials. Int. J. Plast. 16, 179198.

Kocks, U.F., Canova, G.R., 1981. In: Deformations of Polycrystals: Mechanisms and Microstructures. RIS $\emptyset$ National Laboratry, Roskilde, Denmark, p. 35.

Kothari, M., Anand, L., 1998. Elasto-viscoplastic constitutive equations for polycrystalline metals: applications to tantalum. J. Mech. Phys. Solids 46, 51-83.

Kuchnicki, S.N., Cuitino, A.M., Radovitzky, R.A., 2006. Efficient and robust constitutive integrators for single-crystal plasticity modeling. Int. J. Plast. 22, 1988-2011.

Kyparisis, J., 1986. Uniqueness and differentiability of solution of parametric nonlinear complementarity problem. Math. Prog. 36, $105-113$.

Lee, M.G., Lim, H., Adams, B.L., Hirth, J.P., Wagoner, R.H., 2010. A dislocation density-based single crystal constitutive equation. Int. J. Plast. 26, 925-938.

Lemaitre, J., Chaboche, J.L., 1985. Mécanique des matériaux solides. Dunod.

Lim, H., Lee, M.G., Kim, J.H., Adams, B.L., Wagoner, R.H., 2011. Simulation of polycrystal deformation with grain and grain boundary effects. Int. J. Plast..

Ling, X.W., Horstemeyer, M.F., Potirniche, G.P., 2005. On the numerical implementation of 3D rate-dependent single crystal plasticity formulations. Int. J. Numer. Methods Eng. 63, 548-568.

Mandel, J., 1965. Generalisation de la theorie de la plasticite de WT. Koiter. Int. J. Solids Struct. 1, $273-295$.

Maniatty, A.M., Dawson, P.R., Lee, Y.S., 1992. A time integration algorithm for elastoviscoplastic cubic-crystals applied to modeling polycrystalline deformation. Int. J. Numer. Methods Eng. 35, 1565-1588.

Mathur, K.K., Dawson, P.R., 1989. On modeling the development of crystallographic texture in bulk forming processes. Int. J. Plast. 5, 67-94.

McGinty, R.D., McDowell, D.L., 2006. A semi-implicit integration scheme for rate independent finite crystal plasticity. Int. J. Plast. $22,996-1025$.

Megiddo, N., Kojima, M., 1977. On the existence and uniqueness of solutions in nonlinear complementarity theory. Math. Prog. 12, $110-130$.

Miehe, C., Schröder, J., 2001. A comparative study of stress update algorithms for rate-independent and rate-dependent crystal plasticity. Int. J. Numer. Methods Eng. 50, 273-298.

Miehe, C., Schröder, J., Schotte, J., 1999. Computational homogenization analysis in finite plasticity - simulation of texture development in polycrystalline materials. Comput. Method Appl. M 171, 387-418.

Montheillet, F., Gilormini, P., Jonas, J.J., 1985. Relation between axial stresses and texture development during torsion testing: a simplified theory. Acta Metall. 33, 705-717.

Nemat-Nasser, S., Amirkhizi, A.V., 2007. A framework for numerical integration of crystal elasto-plastic constitutive equations compatible with explicit finite element codes. Int. J. Plast. 23, 1918-1937.

Peeters, B., Seefeldt, M., Van Houtte, P., Aernoudt, E., 2001. Taylor ambiguity in BCC polycrystals: a non-problem if substructural anisotropy is considered. Scripta Mater 45, 1349-1356.

Peirce, D., Asaro, R., Needleman, A., 1982. An analysis of nonuniform and localized deformation in ductile single crystals. Acta Metall. 30, 1087-1119.

Peirce, D., Asaro, R., Needleman, A., 1983. Material rate dependence and localized deformation in crystalline solids. Acta Metall. $31,1951-1983$.

Pourboghrat, F., Zamiri, A., Barlat, F., 2007. An effective computational algorithm for rate-independent crystal plasticity based on a single crystal yield surface with an application to tube hydroforming. Int. J. Plast. 23, 1126-1147.

Renouard, M., Wintenberger, M., 1981. Détermination de l'amplitude des glissements dans la déformation plastique homogène d'un monocristal sous l'effet de contraintes et de déplacements imposés. C.R. Acad. Sci. Paris B292, 385-388.

Rossiter, J., Brahme, A., Simha, M.H., Inal, K., Mishra, R., 2010. A new crystal plasticity scheme for explicit time integration codes to simulate deformation in 3D microstructures: Effects of strain path, strain rate and thermal softening on localized deformation in the aluminum alloy 5754 during simple shear. Int. J. Plast. 26, 1702-1725.

Schmid, E., Boas, W., 1935. Plasticity of Crystals. Chapman and Hall, London.

Schmidt-Baldassari, M., 2003. Numerical concepts for rate-independent single crystal plasticity. Comput. Method Appl. M 192, 1261-1280.

Schröder, J., Miehe, C., 1997. Aspects of computational rate-independent crystal plasticity. Comp. Mater. Sci. 9, 168-176.

Simo, J.C., Vu-Quoc, L., 1986. A three dimensional finite strain rod model. Part II: Computational aspects. Comput. Method Appl. M 58, 79-116.

Skalli, A., Fortunier, R., Fillit, R., Driver, J.H., 1985. Crystal rotations during the rolling of large-grained aluminium sheet. Acta Metall., $997-1007$.

Taylor, G., 1938a. Analysis of plastic strain in a cubic crystal. In: Lesels, J.M. (Ed.), Stephen Timoshenko 60th Anniversary Volume.

Taylor, G., 1938b. Plastic strain in metals. J. Inst. Metals 62, 307-324.

Taylor, G.I., Elam, C.F., 1923. The distortion of an aluminum crystal during a tensile test. Proc. Roy. Soc. A $102,643-667$. 
Toth, L.S., Van Houtte, P., A., V.B., 1991. Analytical representation of polycrystal yield surfaces. In: Boehler, J.P., Khan, A.S. (Eds.), Plasticity '91: Third International Symposium on Plasticity and its Current Applications, London, pp. 183-186.

Van Houtte, P., 1981. Adaption of the Taylor theory to the typical substructure of some cold-rolled fcc metals. In: S. Nagashima, E. (Ed.), ICOTOM 6, The Iron and Steel Institute of Japan, pp. 428-437.

Van Houtte, P., 1987. Calculation of the yield locus of textured polycrystals using the Taylor and the relaxed Taylor theories. Textures Microstruct. 7, 29-72.

Watanabe, I., Setoyama, D., Iwata, N., Nakanishi, K., 2010. Characterization of yielding behavior of polycrystalline metals with single crystal plasticity based on representative characteristic length. Int. J. Plast. 26, 570-585.

Zamiri, A.R., Pourboghrat, F., 2010. A novel yield function for single crystals based on combined constraints optimization. Int. J. Plast. 26, 731-746. 